4 Laure Nicolas Annick Ries ${ }^{a^{\star} \#}$, Patricia Alves de Castro $^{\mathrm{b}}$, Lilian Pereira Silva ${ }^{\mathrm{b}}$, 5 Clara Valero ${ }^{b}$, Thaila Fernanda dos Reis ${ }^{b}$, Raquel Saborano ${ }^{c}$, Iola F. Duarte ${ }^{d}$,

6 Gabriela Felix Persinotie ${ }^{e}$ Jacob L. Steenwyk ${ }^{\dagger}$, Antonis Rokas ${ }^{\dagger}$, Fausto Almeida ${ }^{a}$, 7 Jonas Henrique Costa ${ }^{g}$, Taicia Fill ${ }^{g}$, Sarah Sze Wah Wong ${ }^{\text {h }}$, Vishukumar 8 Aimanianda ${ }^{\mathrm{h}}$, Fernando José Santos Rodrigues ${ }^{\mathrm{i}, \mathrm{j}}$, Relber A. Gonçales ${ }^{\mathrm{i}, \mathrm{j}}$, Cláudio 9 Duarte-Oliveira ${ }^{\mathrm{i}, \mathrm{j}}$, Agostinho Carvalho ${ }^{\mathrm{i}, \mathrm{j}}$, Gustavo H. Goldman ${ }^{\mathrm{bH}}$

\section{Aspergillus fumigatus acetate utilisation impacts virulence traits and} pathogenicity 
23 9Instituto de Química, Departamento de Química Orgânica, Universidade de

24 Campinas, Campinas, São Paulo, Brazil

$25{ }^{\mathrm{h}}$ Molecular Mycology Unit, Institut Pasteur, CNRS, UMR2000, Paris, France

26 'Life and Health Sciences Research Institute (ICVS), School of Medicine,

27 University of Minho, Braga, Portugal

'ICVS/3B's - PT Government Associate Laboratory, Guimarães/Braga, Portugal

Running title: Aspergillus fumigatus acetate utilisation

\#Address correspondence to LNA Ries, rieslaure13@gmail.com and GH

*Present address: MRC Centre for Medical Mycology, University of Exeter, 


\section{Abstract}

Aspergillus fumigatus is a major opportunistic fungal pathogen of immunocompromised and immunocompetent hosts. To successfully establish an infection, A. fumigatus needs to use host carbon sources, such as acetate, present in the body fluids and peripheral tissues. However, utilisation of acetate as a carbon source by fungi in the context of infection has not been investigated. This work shows that acetate is metabolised via different pathways in A. fumigatus and that acetate utilisation is under the regulatory control of a transcription factor (TF), FacB. A. fumigatus acetate utilisation is subject to carbon catabolite repression (CCR), although this is only partially dependent on the TF and main regulator of CCR CreA. The available extracellular carbon source, in this case glucose and acetate, significantly affected $A$. fumigatus virulence traits such as secondary metabolite secretion and cell wall composition, with the latter having consequences for resistance to oxidative stress, to anti-fungal drugs and to human neutrophil-mediated killing. Furthermore, deletion of $f a c B$ significantly impaired the in vivo virulence of $A$. fumigatus in both insect and mammalian models of invasive aspergillosis. This is the first report on acetate utilisation in A. fumigatus and this work further highlights the importance of available host-specific carbon sources in shaping fungal virulence traits and subsequent disease outcome, and a potential target for the development of anti-fungal strategies. 
Aspergillus fumigatus is an opportunistic fungal pathogen in humans. During infection, $A$. fumigatus is predicted to use host carbon sources, such as acetate, present in body fluids and peripheral tissues, to sustain growth and promote colonisation and invasion. This work shows that $A$. fumigatus metabolises acetate via different pathways, a process that is dependent on the transcription factor FacB. Furthermore, the type and concentration of the extracellular available carbon source were determined to shape

A. fumigatus virulence determinants such as secondary metabolite secretion and cell wall composition. Subsequently, interactions with immune cells are altered in a carbon sourcespecific manner. FacB is required for $A$. fumigatus in vivo virulence in both insect and mammalian models of invasive aspergillosis. This is the first report that characterises acetate utilisation in $A$. fumigatus and highlights the and potentially subsequent disease outcome. 


\section{Introduction}

Aspergillus fumigatus is a saprotrophic filamentous fungus and opportunistic pathogen of immunocompetent and immunocompromised hosts. Together with other opportunistic fungal pathogens, such as Candida albicans and Cryptococcus neoformans, globally they kill in excess of 1.5 million people a year(1). The severity of the diseases related to A. fumigatus depend on preexisting infections as well as on the status of the host immune system (2). To successfully colonise and survive within the human host, $A$. fumigatus needs to acquire and metabolise nutrients. Essential nutrients include minerals such as iron, copper and zinc, which are required in small amounts; while carbon and nitrogen, the main energy sources for sustaining biosynthetic processes, must be obtained in large quantities(3). Iron, zinc and copper acquisition and metabolism have been studied in A. fumigatus in the context of virulence(4-6), whereas less is known about carbon source acquisition and metabolism in this fungus during infection. Studies have inferred that glucose, lactate and acetate are carbon sources available to fungi in vivo with their availability and concentration depending on the host niche $(7,8)$. Whereas glucose utilisation has been shown to be important for $A$. fumigatus disease progression (9), the utilisation of the physiologically relevant short chain fatty acids (SCFAs) lactate and acetate remain unexplored in this fungus. Indeed, acetate was detected in bronchoalveolar lavage (BAL) samples of healthy and immunosuppressed mice, suggesting the presence of this carbon source, independent of the underlying immune condition, at the A. fumigatus primary site of infection. Our work therefore aimed at characterising $A$. fumigatus acetate utilisation and its relevance for virulence. 
116 In the human body, acetate is present in the blood plasma at concentrations

117 ranging from 0.074 to $0.621 \mathrm{mM}$ depending on the type of artery, diet and 118 alcohol intake(10). Peripheral tissues can consume acetate from the blood stream and oxidise it(10). The main producer of plasma acetate is the gastrointestinal (GI) tract-resident microbiome, with the majority of Gl-resident bacterial species being capable of producing acetate(10). Furthermore, acetate has immunoregulatory properties. Acetate is an agonist for the G-protein coupled receptors (GPCRs) FFA2, FFA3 and GPR109A, which are expressed in a number of immune cells, thus affecting the production of cytokines, the regulation of downstream anti- and pro-inflammatory responses, and recruitment of immune cells(11). Acetate is likely important during invasive fungal infections as it can regulate immunity at distal sites, including the lungs.

As mentioned above, small quantities of acetate were detected in healthy and immunosuppressed mice (8). The lungs are lined with a mucosa and contain a microbiome that has been shown to suffer alterations in the presence of disease(12). The lung microbiome, just like the gut microbiome, may contribute to the production and secretion of SCFAs(13). Studies investigating the production of SCFAs and other molecules by the lung microbiota are nonexistent, probably due to the lungs having been thought of as sterile until a few years ago(12).

Our understanding of the utilization of potential food sources during infection mainly relies on in vitro transcriptional studies(14-16). Despite several studies having investigated $A$. fumigatus gene expression during in vivo infection of chemotherapeutic mice models of invasive aspergillosis, none of these studies have characterised the modulation of genes encoding components required for 
141 carbon source utilisation (17-20). The genome of $A$. fumigatus encodes the acetyl-CoA synthetases (ACS) FacA (Afu4g11080) and PcsA (Afu2g07780), with facA shown to be up-regulated in conidia exposed to neutrophils, and the corresponding protein induced by heat shock and repressed during hypoxic conditions $(14,21,22)$. In $A$. fumigatus, the homologue of the $A$. nidulans transcription factor (TF)-encoding $f a c B$ gene is up-regulated when conidia are exposed to neutrophils(14). Furthermore, $A$. fumigatus conidia which were exposed to human neutrophils from healthy or CGD (chronic granulomatous disease) donors, showed an up-regulation of genes encoding enzymes involved in the glyoxylate cycle, gluconeogenesis, peroxisome function and fatty acid degradation(14), suggesting an induction of metabolic pathways that are required for the utilisation of alternative, non-preferred carbon sources. Together, the aforementioned studies suggest that the utilisation of alternative carbon sources such as SCFAs is important for $A$. fumigatus infection.

Acetate utilisation has been investigated in detail in the model fungus $A$. nidulans. In $A$. nidulans, acetate was shown to be transported by the shortchain carboxylate transporters $A c p A$ and $A c p B$, with the former being expressed in germinating conidia and young germlings, and the latter expressed in mycelia(23). The genome of $A$. fumigatus encodes one homologue of both $a c p A$ and $a c p B$ (Afu2g04080), which remains uncharacterised. Once internalised, acetate is converted by ACS to acetyl-CoA, which subsequently is transported into the mitochondria where it enters the TCA cycle for the synthesis of ATP molecules(24). Furthermore, acetate, in the acetyl-coA form, is oxidised via the glyoxylate cycle and required during gluconeogenesis(24). In A. nidulans, acetate utilisation is under the control of 
166 the TF FacB, which is transcriptionally induced in the presence of acetate(25).

167 FacB controls the expression of the ACS FacA, of the carnitine acetyltransferases FacC, AcuH and AcuJ, of the succinate/fumarate antiporter AcuL and of the glyoxylate cycle malate synthase $\operatorname{AcuE}(25,26)$. The genome of A. fumigatus encodes homologues of the $A$. nidulans components required for acetate metabolism, although they have not been investigated until now. This work characterised the utilisation of acetate in $A$. fumigatus and highlights the importance of the type of available extracellular carbon source in shaping fungal virulence determinants.

\section{Results}

Acetate is metabolised via the glyoxylate and TCA (tricarboxylic acid) cycles and a precursor for different metabolites. To investigate acetate metabolism in $A$. fumigatus, the metabolic fate of acetate was traced by incubating fungal mycelia with ${ }^{13} \mathrm{C}_{2}$-labelled acetate. The $A$. fumigatus wild-type (WT) strain CEA17 was grown for $16 \mathrm{~h}$ in peptone rich-minimal medium (MM) before undergoing $4 \mathrm{~h}$ of carbon starvation in MM. Subsequently, ${ }^{13} \mathrm{C}_{2}$-labelled acetate was added to the cultures for 5 and 15 min before mycelia were separated from the culture medium and immediately snap-frozen in liquid nitrogen. Following metabolite extraction, $1 \mathrm{D}{ }^{1} \mathrm{H}$ and $2 \mathrm{D}{ }^{1} \mathrm{H}^{-13} \mathrm{C}$ HSQC (heteronuclear single quantum coherence) NMR (nuclear magnetic resonance) spectra were recorded for each sample. The uptake of ${ }^{13} \mathrm{C}_{2}$-acetate by $A$. fumigatus was observed through significant increases in carbon satellite peaks (reflecting ${ }^{1} \mathrm{H}_{-}{ }^{13} \mathrm{C}$ coupling) on both sides of the central acetate singlet $(\delta 1.92$ 
$190 \mathrm{ppm}$ ) in ${ }^{1} \mathrm{H}$ spectra of fungal cell extracts (Figure $1 \mathrm{~A}$, top). To identify 191 metabolites that were directly derived from ${ }^{13} \mathrm{C}_{2}$-labelled acetate and determine 192 their fractional enrichment, ${ }^{1} \mathrm{H}^{13} \mathrm{C}$ HSQC spectra from 5 and 15 min samples were compared to spectra of control cells ( $4 \mathrm{~h}$ starvation, no labelling). The extent of ${ }^{13} \mathrm{C}$-incorporation levels was obtained for each metabolite by dividing the $2 \mathrm{D}$ peak intensity in ${ }^{13} \mathrm{C}$-enriched samples by the peak intensity in the matched control sample (with $1.1 \%$ natural abundance in ${ }^{13} \mathrm{C}$ levels). Results showed that labelled ${ }^{13} \mathrm{C}$ from acetate was incorporated into the amino acids alanine at carbon-2 and carbon-3 (C2 and C3), aspartate at $\mathrm{C} 2$ and $\mathrm{C} 3$ and glutamate at $\mathrm{C} 2, \mathrm{C} 3$ and $\mathrm{C} 4$, the glyoxylate/TCA cycle intermediates citrate at $\mathrm{C} 2$, malate at $\mathrm{C} 2$ and $\mathrm{C} 3$ and succinate at $\mathrm{C} 2$ as well as in the glycolipid and glycoprotein compound $\mathrm{N}$-acetylneuraminate at C11 (Figure 1A, bottom).

These results indicate that acetate is taken up and metabolised via the glyoxylate and TCA cycles in A. fumigatus, which is in agreement with studies in S. cerevisiae (27) and $A$. nidulans (24) and in the line with the metabolism of two carbon compounds as the sole carbon source. Indeed, ${ }^{13} \mathrm{C}$ was incorporated into the amino acid and Krebs cycle intermediate aspartate(28) as well as the amino acids alanine and glutamate. The TCA cycle intermediate oxaloacetate is converted into phosphor-enol-pyruvate (gluconeogenesis), which in turn is a precursor for alanine in a two-step process that involves glutamate. Furthermore, ${ }^{13} \mathrm{C}$ was also enriched in other cellular compounds such as $\mathrm{N}$-acetylneuraminate, which is a sialic acid that is present on cell surface acidic glycoconjugates, and which has been shown to contribute to the phagocytic properties of cells of opportunistic fungal pathogens such as Cryptococcus neoformans (29), Candida albicans (30) and A. fumigatus (31). 
215 Hence, this analysis showed that acetate is metabolised by $A$. fumigatus 216 through multiple pathways. the presence of acetate and ethanol as the sole carbon sources. To determine whether acetate utilisation is controlled by a TF in $A$. fumigatus, as was previously described for $A$. nidulans (25), a TF deletion library (32) was screened for reduced growth on plates containing MM supplemented with $0.5 \%$ $(\mathrm{w} / \mathrm{v})$ acetate $(\mathrm{AMM})$ as the sole carbon source. Several strains were identified, and subsequent confirmation growth experiments, in both solid (radial growth) and liquid (dry weight) AMM, resulted in the selection of 5 strains that had reduced growth in acetate, but presented no growth defects in glucose-rich MM (GMM) (Supplementary Figure 1A-C at 10.6084/m9.figshare.14740482, Figure 1B-D). These strains were deleted for genes acuK (Afu2g05830), acuM (Afu2g12330), facB (Afu1g13510), farA (Afu4g03960) and mtfA (Afu6g02690) (Supplementary Figure 1 at 10.6084/m9.figshare.14740482, Figure 1B-D). AcuM, AcuK and MtfA have been characterised in A. fumigatus and have been shown to be important for alternative carbon source utilisation and virulence(33, 34). Furthermore, farA was shown to be important for fatty acid utilisation and was up-regulated in fungal cells exposed to human neutrophils(14). In contrast, A. fumigatus FacB, which is the homologue of $A$. nidulans FacB, remains uncharacterised. We therefore aimed at further deciphering the role of the TF FacB in $A$. fumigatus acetate utilisation and virulence. FacB was also essential for growth in medium with ethanol as the sole carbon source but not for growth

238 in the presence of different fatty acids (Supplementary Figure 1D at 239 10.6084/m9.figshare.14740482). Ethanol and acetate are two carbon 
240 compounds that require identical metabolic pathways with ethanol being converted to acetate via the metabolic intermediate acetaldehyde(35). Reintroduction of $A$. fumigatus $f a c B$ in the $\triangle f a c B$ background strain at the facB locus through homologous recombination restored growth in acetate (Figure 1BD, Supplementary Figure 1D at 10.6084/m9.figshare.14740482), confirming that the FacB-encoding gene is essential for $A$. fumigatus growth on two carbon compounds.

FacB controls acetate utilisation through regulating genes encoding enzymes required for acetate metabolism. To gain further insight into $A$. fumigatus acetate metabolism and to describe a role of FacB in the control of acetate utilisation, the transcriptional response of the wild-type (WT) and $\triangle \mathrm{facB}$ strains was assessed by RNA-sequencing (RNA-seq), when grown for $24 \mathrm{~h}$ in fructose-rich (control) MM and after transfer for $0.5 \mathrm{~h}$ (short incubation) or $6 \mathrm{~h}$ (long incubation) to MM supplemented with $0.1 \% \mathrm{w} / \mathrm{v}$ (low concentration) or $1 \%$ $w / v$ (high concentration) acetate. We chose different concentrations of acetate and time points in order to decipher the transcriptional response in the presence of abundant and limiting carbon source concentrations after short and prolonged exposure. The number of significantly differentially expressed genes (DEGs) were defined as having a $-1<\log 2 \mathrm{FC}$ (fold change) $<1$ and an adjusted $p$-value $<\quad 0.05$ (Supplementary File $\quad 1$ at 10.6084/m9.figshare.14740482, Table 1). Two comparisons were carried out: i) gene expression in the presence of the four different acetate conditions against gene expression in the control (fructose) condition in the WT strain; and ii) gene expression in the WT strain against gene expression in the $\triangle \mathrm{fac} B$ strain in the presence of the different acetate conditions (Table 1, 8 comparisons in total). 
265 Gene ontology (GO) and Functional Categorisation (FunCat) analyses could not be performed for many of the comparisons shown in Table 1, probably due to a

267 low number of DEGs in some conditions (Table 1). DEGs were therefore manually inspected and divided into the following categories: a) amino acid, protein and nitrogen (urea, nitrate, ammonium) metabolism (degradation and biosynthesis); b) carbohydrate and lipid metabolism, including genes encoding enzymes required for lipid, fatty acid and acetate degradation, CAZymes (carbohydrate active enzymes) and the metabolism of other sugars; c) cell signalling (protein kinases, phosphatases, regulators of G-protein signalling and G-protein coupled receptors - GPCRs); d) cell membrane and cell wall (ergosterol, chitin and glucan biosynthesis/degradation);

e) miscellaneous (genes encoding enzymes with diverse functions that do not fit into the other categories); f) oxidation/reduction and respiration (oxidoreductases, monooxygenases and respiratory chain enzymes); g) secondary metabolism; h) transcription factors; i) transporters (sugars, amino acids, ammonium, nitrate, ions, metals and multidrug); j) unknown (gene encoding proteins with unknown/uncharacterised functions) and k) putative virulence factors (proteases and proteins important for adhesion and interaction with the extracellular environment) (Supplementary Files 2 and 3 at 10.6084/m9.figshare.14740482; Supplementary Figure 2 at 10.6084/m9.figshare.14740482). In the WT strain, this categorisation was carried out for all DEGs with a $-3<\log 2 \mathrm{FC}<3$ in order to identify genes with the highest differential expression pattern. For comparisons between the WT and $\triangle \mathrm{facB}$ strains, categorisation was carried out for all DEGs with a $-1.5<\log 2 \mathrm{FC}<1.5$ in order to include as many DEGs as possible. 
290 The majority of DEGs (34 - 46\%) encoded proteins of unknown function, whereas genes encoding enzymes required for carbohydrate and carbon compound (CC) metabolism, oxidation/reduction and respiration, secondary metabolism and transporters constituted 38 - 48\% of all DEGs (Supplementary Figure 2 at 10.6084/m9.figshare.14740482) suggesting the presence of acetate influences the regulation of these processes. No particular enrichment for any of the aforementioned categories was found for the here studied conditions

297 (Supplementary Figure 2 at 10.6084/m9.figshare.14740482). There were differences though in the type of secondary metabolites (SMs), transporters as well as respiratory and carbon source metabolism encoded by the DEGs (Supplementary Figure 2 at 10.6084/m9.figshare.14740482).

To further unravel the role of FacB in acetate utilisation, we focused on DEGs that encode enzymes important for acetate metabolism. In the wild-type strain, genes encoding the ACS FacA (but not the ACS PcsA), a carnitine acetyl transferase (Afu1g12340), a mitochondrial carnitine:acyl carnitine carrier (Afu6g14100), the isocitrate lyase (ICL) AcuD (acuD, glyoxylate cycle) and the malate synthase AcuE (acuE, glyoxylate cycle) were highly expressed in all acetate conditions; in contrast, these genes were repressed in the $\Delta f a c B$ strain (Figure 2A). The exception was in the presence of $6 \mathrm{~h}$ in $0.1 \% \mathrm{w} / \mathrm{v}$ acetate, where these genes were not expressed in the WT strain but they were induced in the $f a c B$ deletion strain (Figure $2 A$ ). This is likely due to carbon starvation, which would occur in these conditions. Assessment of the expression of facA, Afu1g12340, Afu6g14100 and $a c u D$ by qRT (real-time reverse transcriptase)PCR in the same conditions confirmed the RNA-seq data (Figure 2B, 2C). 
314 To further confirm the transcriptional data, we assayed the activities of ACS and ICL (isocitrate lyase) in the WT, $\triangle \mathrm{facB}$ and $\triangle \mathrm{facB}:$ :facB $B^{+}$strains when grown in the presence of $1 \% \mathrm{w} / \mathrm{v}$ acetate for $0.5 \mathrm{~h}, 6 \mathrm{~h}$ and $22 \mathrm{~h}$. In agreement with the RNA-seq data, ACS and ICL activities were induced in the presence of acetate in the WT and $\triangle f a c B:: f a c B^{+}$strains. No significant difference in ACS and ICL activities were observed between the WT and $\Delta f a c B:: f a c B^{+}$strains, whereas these enzyme activities were significantly reduced in the $\Delta$ facB strain in all tested conditions (Figure 2D). ICL activity was completely dependent on FacB with the loss of facB resulting in no enzyme activity (Figure 2D). In contrast, ACS activity was reduced $\sim 20-30 \%$ in the $\triangle f a c B$ strain when compared to the WT and $\triangle \mathrm{facB}:$ :facB ${ }^{+}$strains (Figure 2D). The observed ACS activity is likely due to the activity of the second $A$. fumigatus ACS PcsA. Our RNA-seq data shows that $p c s A$ is not under the regulatory control of FacB in the here tested conditions, whereas the expression of the single ICL-encoding gene $a c u D$, is regulated by FacB (Figure $2 \mathrm{~A}$ ). These results suggest that FacB controls acetate utilisation through regulating genes encoding enzymes required for acetate metabolism.

Acetate metabolism is subject to carbon catabolite repression (CCR). $\ln A$. fumigatus, CCR is a cellular process which directs primary metabolism to the utilisation of preferred carbon sources (glucose) and results in the repression of genes required for the utilisation of alternative carbon sources (acetate)(36). The opportunistic yeast pathogen $C$. albicans is able to simultaneously use glucose and lactate, due to the loss of an ubiquitination site on ICL(37). This increased metabolic flexibility plays a role in the adaptation of $C$. albicans to the host environment with the addition of an ubiquitination site to $C$. albicans ICL 
339 resulting in decreased resistance to phagocytosis by macrophages, decreased 340 fungal burden in the GI tract, and decreased dissemination to the kidneys(38).

341 To determine whether $A$. fumigatus is able to use glucose and acetate simultaneously, transcriptional and enzymatic studies were performed in the WT and $\triangle f a c B$ strains in the presence of equimolar concentrations of glucose and acetate. We also included a strain deleted for the TF CreA, which is a transcriptional regulator of CCR (36). Strains were grown in the presence of $12.2 \mathrm{mM}(0.1 \% \mathrm{w} / \mathrm{v})$ and $122 \mathrm{mM}(1 \% \mathrm{w} / \mathrm{v})$ acetate without or with equimolar concentrations of glucose for $0.5 \mathrm{~h}$, before expression of genes $f a c B, \operatorname{fac} A$, Afu1g12340, Afu6g14100 and acuD were determined by qRT-PCR (Figure 3AB). The presence of the different concentrations of glucose caused a significant down-regulation of all genes, except for facB in the presence of $122 \mathrm{mM}$ acetate and glucose (Figures $3 A-B$ ). It is possible that the expression of $f a c B$ is dependent on the concentration of the externally available carbon source. In Aspergillus spp., high and low affinity carbon source transporters are expressed depending on the concentration of the extracellular carbon source (39). A similar scenario can be envisaged for transcription factors (TFs), especially as they respond to external stimuli, with some TFs being highly induced under nutrient limiting conditions and repressed in nutrient sufficient conditions (20). Alternatively, Aspergillus transcriptions factors are not always regulated at the transcriptional level as previously shown (40). These results suggest that $A$. fumigatus acetate metabolism is subject to CCR as has been described in $A$. nidulans(25, 26).

In the presence of $12.2 \mathrm{mM}$ acetate, deletion of creA caused a significant downregulation of $f a c A, A f u 6 g 14100$ and $a c u D$; whereas in the simultaneous 
364 presence of $12.2 \mathrm{mM}$ acetate and glucose, the absence of cre $A$ significantly increased facB and Afu6g14100 gene expression (Figure 3A). In the presence of $122 \mathrm{mM}$ acetate, deletion of creA significantly reduced Afu1g12340 and Afu6g14100 gene expression, whereas upon in the presence of glucose, the expression of all genes, except for $f a c B$, was increased, although not to WT levels (Figure 3B). The exception was the expression of acuD in the $\triangle c r e A$ strain in the presence of $122 \mathrm{mM}$ acetate and glucose, which was similar to the expression levels of $a c u D$ in the WT strain in the presence of $122 \mathrm{mM}$ acetate (Figure 3B). These results suggest that: i) CreA may be involved in the control of genes required for alternative carbon source utilisation and that ii) acetate metabolism (with the exception of $a c u D$ ) is partially dependent on CreAmediated CCR in a concentration-dependent manner.

Next ACS and ICL activities were measured in the presence of $122 \mathrm{mM}$ acetate and glucose after $0.5 \mathrm{~h}, 6 \mathrm{~h}$ and $22 \mathrm{~h}$. Enzyme activities were lower in the presence of acetate and glucose (Figure 3C) than in the presence of acetate only (Figure 2C), supporting the observed transcriptional repression of the corresponding genes in the presence of glucose. Basal ACS activity was detected in all conditions (likely due to the presence of the FacB-dependent ACS FacB and the FacB-independent ACS PcsA) whereas ICL activity was not detected at $0.5 \mathrm{~h}$ and $6 \mathrm{~h}$. This is in agreement with the transcriptional data, suggesting that ICL activity is completely dependent on FacB for induction (Figures 2B-C, 3B-C) and CreA for repression (Figure 3B). After $22 \mathrm{~h}$ incubation in both carbon sources, enzyme activities increased, which may be due to low glucose concentrations ( 30\%) in the culture medium (Figure 3D), making acetate the predominant available carbon source. Furthermore, significantly 
more extracellular glucose was present in supernatants of the $\triangle \mathrm{facB}$ strain (Figure 3D), suggesting that FacB may also be involved in the utilisation of other carbon sources. Together, these results suggest that genes and enzymes involved in acetate utilisation are subject to CCR and that their regulation is partially controlled by CreA.

\section{The extracellular carbon source influences the levels of secreted} secondary metabolites (SMs). The secretion of SMs has been shown to be essential for $A$. fumigatus proliferation within the natural environment and mammalian host, for evasion and modulation of the host immune system and for virulence(41). Our RNA-seq data shows that many DEGs are part of the fumagillin, pseurotin A, pyomelanin and gliotoxin SM biosynthetic gene clusters (BGCs) (Supplementary Figure 2 at 10.6084/m9.figshare.14740482, Figure 4AB). SM BGCs are mainly expressed in the WT strain in the presence of $1 \% \mathrm{w} / \mathrm{v}$ acetate or after $6 \mathrm{~h}$ incubation in MM supplemented with $0.1 \% \mathrm{w} / \mathrm{v}$ acetate (Figure 4A-B). In contrast, these DEGs have reduced expression or are repressed in the in the $\triangle f a c B$ strain (Figure $4 A-B$ ). Furthermore, the expression profiles of these genes were often reversed between the WT and $\Delta f a c B$ strains in these conditions, suggesting that the metabolic pathways regulated by FacB are important for SM gene expression.

To determine whether SMs are secreted specifically in the presence of acetate and dependent on FacB, high performance liquid chromatography (HPLC) was performed on culture supernatants from the WT and $\triangle f a c B$ strains grown for 24 $\mathrm{h}$ in fructose-rich $\mathrm{MM}$ and after transfer to $\mathrm{MM}$ supplemented with $0.1 \% \mathrm{w} / \mathrm{v}$ and $1 \% \mathrm{w} / \mathrm{v}$ acetate for $24 \mathrm{~h}$. This pre-growth in fructose ensured that the starting biomass was similar for all samples. In addition, SM profiles were examined for 
414 the WT strain when grown in the same conditions, with the exception that acetate was replaced with glucose as the main carbon source. After $24 \mathrm{~h}$, a total of 18 SMs including the previously characterised $(42,43)$ fumiquinazolines $A$ and $D$, fumitremorgin $C$, pyripyropene $A$, pseurotins $A$ and $F 2$, fungisporin and brevianamide $\mathrm{F}$, were identified in culture supernatants from strains grown in all conditions (Supplementary Table 2 at 10.6084/m9.figshare.14740482). We did not detect gliotoxin or pyomelanin in culture supernatants.

Subsequently, the concentrations of characterised SMs were quantified to determine whether the extracellular available carbon source influences the levels of secreted SMs. In the WT strain, concentrations of all these SMs, with the exception of fumiquinazoline and fungisporin, were significantly higher in the presence of $1 \% \mathrm{w} / \mathrm{v}$ glucose than in the presence of $1 \% \mathrm{w} / \mathrm{v}$ acetate (Figure $4 C)$. Similarly, in the presence of $0.1 \% \mathrm{w} / \mathrm{v}$ glucose, secreted levels of brevianamide $\mathrm{F}$, fumiquinazoline, fumitremorgin $\mathrm{C}$ and pseurotin $\mathrm{A}$ were significantly higher when compared to concentrations in the presence of $0.1 \%$ $w / v$ acetate; whereas levels of fumagillin and fungisporin A were significantly higher in the presence of $0.1 \% \mathrm{w} / \mathrm{v}$ acetate than in the presence of $0.1 \%$ glucose (Figure 4C). Furthermore, differences in levels of secreted SMs were also seen between the two different concentrations of the same carbon source (Figure $4 C$ ). In addition, deletion of $f a c B$ resulted in a significant decrease in concentrations of secreted SMs in the presence of different concentrations of extracellular acetate with the exception of fumagillin (Figure 4C). extracellular carbon source affects the levels of secreted SMs. 
438 The composition of the A. fumigatus cell wall is carbon source dependent.

439 In $A$. fumigatus, the composition of the culture medium influenced cell wall 440 composition, thus modulating their sensitivity to antifungal agents(44). In addition, primary carbon metabolism was shown to influence cell wall content and/or organisation(45). To investigate whether glucose and acetate, respectively representing preferred and alternative carbon sources would also influence cell wall composition, we determined the quantities of cell wall polysaccharides in the $A$. fumigatus WT strain when grown in the presence of each of these carbon sources.

Cell wall alkali-insoluble (AI) and alkali-soluble (AS) fractions were prepared of WT mycelia grown for $24 \mathrm{~h}$ in MM supplemented with $1 \% \mathrm{w} / \mathrm{v}$ glucose or acetate and analysed by gas-liquid chromatography (Supplementary Figure 3A at $10.6084 / \mathrm{m} 9$. figshare.14740482). Results show that there is a significant increase in the percentage of the cell wall Al fraction in the presence of acetate due to increased concentrations of glucose ( $\beta-1,3$-glucan) and glucosamine

453 (chitin) (Figures 5A and 5B; Supplementary Figure $3 \mathrm{~A}$ at $45410.6084 / \mathrm{m9}$.figshare.14740482). In contrast, the percentage of cell wall AS fraction was significantly reduced in the presence of acetate predominantly due to decreased levels of glucose ( $\alpha-1,3-$ glucan), although significantly decreased

457 levels of mannose and galactose were also observed (Figure 5A,B; 458 Supplementary Figure $3 \mathrm{~A}$ at $10.6084 / \mathrm{m} 9$.figshare.14740482). These results suggest that the type of extracellular carbon source significantly influences $A$. fumigatus cell wall composition and organisation.

461 Oxidative stress and antifungal drug tolerance are carbon source- 
463 during infection as it represents the main line of defence for the fungus and is responsible for interacting with and modulating host immune cells as well as for oxidative stress and antifungal drug resistance(46). The aforementioned results show that the type of carbon source has an effect on cell wall polysaccharide concentrations. Subsequently, oxidative stress and antifungal drug resistance were determined in the $A$. fumigatus WT when grown in the presence of glucose or acetate.

First, the WT strain was grown in the presence of GMM or AMM supplemented with the oxidative stress-inducing compounds hydrogen peroxide $\left(\mathrm{H}_{2} \mathrm{O}_{2}\right)$, menadione and $t$-butyl hydroperoxide, before colony diameters were measured and the percentage of growth was normalised by the growth in the control, drugfree condition for each carbon source. Growth was significantly reduced in the presence of AMM supplemented with the oxidative stress-inducing compounds when compared to growth in the presence of GMM supplemented with the oxidative stress-inducing compounds (Figure 5C, Supplementary Figure 3B at 10.6084/m9.figshare.14740482). These results suggest that the presence of acetate increases sensitivity to oxidative stress in $A$. fumigatus.

Next, we determined resistance to antifungal drugs, including different azoles, amphotericin B and caspofungin, when the WT strain was grown in the presence of glucose and acetate. We performed MIC (minimal inhibitory concentration) assays of amphotericin $\mathrm{B}$, voriconazole, itraconazole and posaconazole when $A$. fumigatus was grown in RPMI medium (standard reference medium used for MIC assays), GMM and AMM for 72 h. A. fumigatus grown in the presence of $A M M$, was slightly more susceptible to amphotericin B than when compared to growth in the presence of RPMI and GMM (Table 2). 
$488 \quad$ No difference in susceptibility was observed for the different here tested azoles when compared to RPMI although reduced growth in the presence of these azoles was observed when comparing MIC between GMM and AMM (Table 2). The WT strain was also grown in GMM or AMM supplemented with increasing concentrations of the echinocandin and second line therapy drug caspofungin (47). In the presence of 0.5 and $2 \mu \mathrm{g} / \mathrm{ml}$ caspofungin, growth was severely inhibited and did not differ between both carbon sources (Figure 5D, Supplementary Figure $3 \mathrm{C}$ at 10.6084/m9.figshare.14740482). At $8 \mu \mathrm{g} / \mathrm{ml}$ caspofungin, the WT strain had increased growth in the presence of glucose and acetate, due to the caspofungin paradoxical effect (increased fungal growth in the presence of higher caspofungin concentrations (48)). In the presence of acetate, the WT was less able to recover growth when compared to growth on GMM (Figure 5D, Supplementary Figure 3C at 10.6084/m9.figshare.14740482).

These results suggest that oxidative stress and antifungal drug resistance change depending on the extracellular, available carbon source.

Acetate-grown hyphae are more susceptible to human neutrophilmediated killing than hyphae grown in the presence of glucose. The aforementioned results indicate that growth in the presence of acetate influences virulence determinants such as SM production, cell wall composition, oxidative stress and antifungal drug resistance when compared to growth in the presence of energetically more favourable carbon sources such as glucose. To determine the role of carbon source-mediated growth for resistance against human neutrophils, we assayed the viability of hyphae, pre-grown in MM 511 supplemented with either glucose or acetate as the sole carbon source. To ensure that a similar number of conidia had germinated prior to incubation with 
513 neutrophils, microscopy was performed and the number of germinated conidia was counted. After incubation for $8 \mathrm{~h}$ in GMM and $13 \mathrm{~h}$ in $\mathrm{AMM}, \sim 90 \%$ of conidia had germinated in both conditions (Supplementary Figure 3D at 10.6084/m9.figshare.14740482) and they were visually inspected to be similar

517 in length (data not shown). Human neutrophils used at different multiplicity of 518 infection (MOI), killed significantly more (60 - 80\%) A. fumigatus hyphae grown in AMM than when compared to hyphae (50 - 60\%) pre-grown in GMM (Figure $5 \mathrm{E})$. These results indicate that hyphae grown in acetate-rich medium are more susceptible to human neutrophil-mediated killing than hyphae grown in the presence of glucose.

FacB is crucial for virulence in insect and murine models of disseminated and invasive pulmonary aspergillosis (IPA). Lastly, we assessed the virulence of the $\triangle f a c B$ strain in vitro and in vivo. First, the capacity of murine bone marrow-derived macrophages (BMDM) to phagocytose and kill WT, $\triangle$ facB and $\triangle f a c B:: f a c B^{+}$conidia was determined. The $\triangle f a c B$ strain was significantly more susceptible to BMDM phagocytosis (Fig. 5F) and a significantly higher amount of $\Delta f a c B$ conidia were killed in comparison to the WT and $\Delta f a c B:: f a c B^{+}$ strains (Fig. 5G).

Next, virulence of the WT, $\triangle \mathrm{facB}$ and $\triangle \mathrm{facB}:: \mathrm{facB}^{+}$strains was determined in the wax moth Galleria mellonella and in a neutropenic murine model of IPA. We used different animal models as virulence $A$. fumigatus was shown to be dependent on the status of the host immune system(49). In G. mellonella (Figure $6 \mathrm{~A}$ ) and in chemotherapeutic mice (Figure $6 \mathrm{~B}$ ), the $\triangle \mathrm{facB}$ strain was hypovirulent when compared to the WT and $\triangle \mathrm{facB}:$ :fac $B^{+}$strains. In the insect model, the WT and $\triangle \mathrm{facB}:: \mathrm{fac}^{+}$strains killed all larvae after 8 days, whereas 
$53880 \%$ of larvae infected with the $\Delta$ facB strain survived after 10 days (Figure $6 \mathrm{~A}$ ).

539 Similarly, the WT and $\triangle \mathrm{facB}:: \mathrm{facB}^{+}$strains killed all mice after 4 days, whereas

$54010 \%$ of mice infected with the $\triangle f a c B$ strain survived 6 days post-infection (p.i)

541 (Figure 6B). In agreement, fungal burden was significantly reduced for the

$542 \Delta$ facB strain after 3 (Figure $6 \mathrm{C}$ ) and 7 (Figure 6D) days p.i. in murine lungs

543 when compared to the WT and $\Delta$ facB::facB ${ }^{+}$strains. In addition, histopathology

544 analyses of murine lungs after 3 p.i., showed significantly reduced inflammation

545 (Figure 6E, F) and growth in the lungs (Figure 6F) for the $\Delta$ facB strain after 3 days p.i. when compared to the WT and $\triangle \mathrm{facB}:: \mathrm{facB}^{+}$strains. Together these

547 results suggest that FacB is important for $A$. fumigatus virulence in insect and 548 mammalian hosts.

549 To determine whether the observed reduction in virulence of the $\mathrm{facB}$ deletion strain may be due to growth defects, the WT, $\triangle$ facB and $\triangle \mathrm{facB}:: \mathrm{facB}^{+}$strains were grown for $72 \mathrm{~h}$ in the presence of different media that are similar to the mammalian host environment before fungal biomass was quantified. The $\Delta$ facB strain had significantly reduced growth in the presence of low and high-glucose containing DMEM (Dulbecco's Modified Eagle's Medium), FBS (fetal bovine serum) and beef extract when compared to the WT and $\triangle \mathrm{facB}:$ :facB $B^{+}$strains but not in the presence of RPMI 1640 medium and minimal medium supplemented with glucose (control) (Supplementary Figure 3E at 558 10.6084/m9.figshare.14740482). These results suggest that the observed 559 reduction in virulence of the $\triangle f a c B$ strain is at least partially due to strainspecific growth defects in a mammalian host environment. 
562

563

564

565

566

567

568

569

570

571

572

573

574

575

576

577

578

579

580

581

582

583

584

585

586

\section{Discussion}

This work aimed at deciphering the regulation of the physiologically relevant carbon source acetate in $A$. fumigatus, and at determining its relevance for fungal virulence. As a first step, we show that $A$. fumigatus can take up and metabolise acetate via the TCA and glyoxylate cycles which is in agreement with studies in S. cerevisiae and $A$. nidulans $(24,27)$. Furthermore, $A$. fumigatus acetate metabolism was shown to be under the regulatory control of the transcription factor $\mathrm{FacB}$, which controls the expression of genes encoding enzymes that are required for the conversion of acetate to acetyl-CoA, for mitochondrial import of acetyl-CoA and enzymes of the glyoxylate cycle. In agreement with the transcriptional data, deletion of facB also affected ACS (conversion of acetate to acetyl-CoA) and ICL (glyoxylate cycle) enzyme activities. These results are in agreement with studies in $A$. nidulans, where acetate utilisation as sole carbon source is also dependent on $\mathrm{FacB}$, with this TF regulating the expression of the ACS-encoding gene facA and the glyoxylate cycle enzyme-encoding genes acuD (ICL) and acuF (malate synthase)(25).

In addition, we show that $A$. fumigatus acetate metabolism-related genes as well as ACS and ICL activities are subject to CCR. This is in contrast to findings in C. albicans where the addition of glucose to lactate-grown cells did not result in CCR (38). It is important to note here that the experimental conditions differed between our study and (38) (e.g. A. fumigatus growth in acetate and glucose versus $C$. albicans growth in lactate then addition of glucose). It is well known that the addition of glucose to cultures causes CCR in Aspergillus spp (36), and the rationale here was therefore to present the fungus with equimolar concentrations of both carbon sources during all growth stages. Acetate 
587 metabolism was also reported to be subject to CCR in $A$. nidulans, with facB 588 and the carnitine acetyltransferase-encoding gene facC being under the 589 regulatory control of the CC-repressor CreA $(50,51)$.

590 In A. fumigatus, CreA-dependent repression of genes encoding enzymes 591 required for acetate metabolism was observed only for the ICL-encoding gene $a c u D$ whereas the other genes were only partially dependent on CreA-mediated repression. A discrepancy between $a c u D$ transcript levels and protein activity was observed. It is possible that basal transcript levels are present in all conditions to allow to quickly respond to changes in extracellular available nutrient sources, but that post-transcriptional processing is not taking place. Indeed, gene transcript levels cannot predict protein levels and activity due to mRNA spatiotemporal fluctuations and availability of protein synthesis components (52). Our data suggests that additional repressor proteins and/or mechanisms exist. In agreement, in $A$. nidulans, CreA has been shown to be part of a protein complex that mediates target gene repression, and that corepressor proteins are crucial for CreA function(53). Furthermore, deletion of $A$. fumigatus creA resulted in significantly decreased expression of acetate utilisation genes in the presence of acetate, suggesting that CreA may be involved in the regulation of these genes in the absence of glucose. In $A$. nidulans, CreA was shown to be important for growth in different carbon, 607 nitrogen and lipid sources and for amino acid metabolism(40); whereas in the filamentous fungus Trichoderma reesei, CRE1 was proposed to have roles in chromatin remodelling and developmental processes and was shown to also act as a transcriptional activator (54). These studies suggest that CreA and its 
611 homologues have additional roles, other than mitigating CCR, in filamentous

612 fungi.

613 The deletion of $f a c B$ caused a significantly differential expression of genes that 614 are part of SM biosynthetic gene clusters (BGC) as well as in secreted SMs. A 615 direct correlation between transcript and secreted protein levels is not possible 616 as gene transcript levels cannot predict concentrations of biosynthesised 617 proteins, due to intrinsic fluctuations in mRNA and availability of protein synthesis components (52). Furthermore, SM BGC regulation is extremely complex and governed by many TFs and epigenetic modifications, which result in the expression of different SM BGC in any given condition (55).

621 Subsequently, not all of these SMs are secreted and transcriptional expression of gliotoxin and pyomelanin BGC, as observed here, may be a consequence of the biosynthesis and secretion of other SMs. The role of FacB in the regulation of SM biosynthesis is perhaps not surprising as this TF regulates genes encoding enzymes of central carbon metabolic pathways (e.g. glyoxylate cycle, shown in this work) during growth on alternative carbon sources. SMs are known to be derived from these central metabolic pathways(55) and this study further emphasises that the amount of produced SMs occurs in a carbon source-dependent manner. Alternatively, the observed low concentrations of secreted SMs of the $\Delta f a c B$ strains may be due to the inability of this strain to grow in the presence of acetate. Notably, SMs measured in the here defined conditions may be secreted in response to carbon starvation, especially in the presence of low $(0.1 \%)$ glucose and acetate conditions. These results further emphasise the role of $\mathrm{FacB}$ in regulating carbon metabolism. In addition, these 
636 depend on host carbon sources and on carbon source starvation, which is encountered within different, nutrient-poor host niches.

\section{8}

Utilisation of different carbon sources also affects the composition of the $A$. fumigatus cell wall, a factor crucial for fungal virulence and pathogenicity and survival within the human host(2). This study shows that growth on acetate results in increased concentrations of the structural polysaccharides $\beta-1,3-$ glucan and chitin and reduced levels of the cementing, "glue-like" $\alpha-1,3$-glucan when compared to the $A$. fumigatus cell wall after growth in glucose. Changes in cell wall composition are likely due to differences in primary carbon metabolism that govern the utilisation of these carbon sources and that generate the cell wall polysaccharide precursors(45). Indeed, impairments in $A$. fumigatus glucose utilisation metabolic pathways resulted in an altered cell wall(45). In agreement with other studies, our work suggests that these changes in cell wall composition influence $A$.

A. fumigatus susceptibility to physiologicalrelevant stresses and antifungal drugs $(44,56)$. Likely, the significant reduction in the cementing $\alpha-1,3$-glucan disturbs the organisation of the other cell wall polysaccharides, increasing fungal cell wall permeability and susceptibility to extracellular stresses. This is true for the $\beta-1,3$-glucan synthase inhibitor caspofungin and for amphotericin B, which physiochemically interacts with membrane sterols (57). In contrast, increased susceptibility of acetate-grown hyphae to azoles, a class of antifungal drugs that impair ergosterol biosynthesis 657 through targeting lanosterol demethylase of the ergosterol biosynthetic pathway, was not observed. A possible explanation for this may be that caspofungin and amphotericin B both target cell wall and cell membrane 
661 differences in cell wall organisation and polysaccharide content, azole uptake is not affected. In agreement with our data where growth on acetate increases sensitivity to oxidative stress-inducing compounds, $A$. fumigatus acetategerminated hyphae were more susceptible to human neutrophil-mediated killing in vitro when compared to hyphae grown in the presence of glucose. The observed increase in susceptibility to different immune cells is probably due to the differences in cell wall composition resulting from growth in both carbon sources. Our observations are in agreement with a previous study, which showed that a hypoxic environment influenced cell wall thickness, composition and surface exposed polysaccharides, subsequently increasing neutrophil and macrophage reactiveness and activity against $A$. fumigatus(58). The physiological significance of the aforementioned acetate-related differences in cell wall composition, antifungal drug and oxidative stress resistance and interaction with neutrophils remains to be determined. Although acetate was shown to present in the BAL of mice (8), we currently do not know how acetate concentrations fluctuate within certain parts of the lung and the surrounding tissues, as has previously been shown for lung hypoxic microenvironments. It will be interesting to study the distribution of carbon sources in different host niches in future studies.

The $\triangle f a c B$ strain exhibited increased susceptibility to macrophage-mediated phagocytosis and killing. Due to the inability of the $\triangle \mathrm{facB}$ strain to grow in the presence of acetate, we were unable to quantify cell wall composition in this strain and therefore determine whether this is a contributing factor when challenged with BMDMs. The inability of the $\triangle f a c B$ strain to use acetate may account for the observed increase in phagocytosis and killing of this strain, 
686

687

688

689

690

691

692

693

694

695

696

697

698

699

700

701

702

703

704

705

706

707

708

709

710

especially as acetate is available as a carbon source in macrophages(59). In agreement, the expression of genes required for acetate utilisation in the presence of macrophages has been observed for $A$. fumigatus and prokaryotic pathogens $(14,60)$. It is unlikely that the observed increased phagocytosis and killing of this strain is due to defects in the glyoxylate cycle as previous studies have revealed that glyoxylate cycle enzymes are dispensable for $A$. fumigatus virulence(61)'(62). This is further supported by our findings that the utilisation of fatty acids, which results in acetyl-CoA production via $\beta$-oxidation(26), is independent of FacB.

Furthermore, the $\triangle f a c B$ strain was hypovirulent in both insect and murine models of invasive aspergillosis. Reduced growth of the facB deletion strain in media simulating the host environment may account for the reduction in virulence observed for this strain. In addition to acetate metabolism, other FacBcontrolled metabolic pathways, which are required for growth in these highly complex nutrient sources may be important for pathogenicity. Investigating the virulence of strains deleted for genes encoding involved in central metabolic pathways such as the phosphoenolpyruvate (PEP) carboxykinase AcuF, the ACS FacA and acetyl-CoA mitochondrial and peroxisome import proteins is subject to future investigations and may further explain the observed reduction in growth. Additional mechanisms may exist, which are important for virulence and are regulated by $\mathrm{FacB}$, especially as the $\mathrm{FacB}$ regulon is large. Our RNAseq data shows that FacB regulates genes encoding proteins important for the production of SMs and oxidoreduction processes, which contribute to virulence. In addition, we cannot rule out that FacB has different targets in vitro when compared to in vivo, as was previously shown for the A. fumigatus AcuM and 
711 AcuK transcription factors that are involved in the regulation of gluconeogenesis

712 and iron acquisition(33). The exact mechanism of FacB for in vivo virulence

713 thus remains to be determined but is possibly a combination of the 714 aforementioned factors.

715 In summary, this study describes acetate utilisation in $A$. fumigatus and 716 highlights the importance of carbon source utilisation and metabolic pathways 717 for determining a variety of fungal traits that are crucial for virulence and that potentially shape disease outcome. Future studies should focus on this neglected area of exploring carbon source variety and availability in host primary sites of infection in order to better understand fungal pathogen nutrient requirements and utilisation, which can potentially be targeted for developing anti-fungal strategies.

Strains and media. All strains used in this study are listed in Supplementary Table 1 at $10.6084 / \mathrm{m} 9$.figshare.14740482. Re-introduction of $\mathrm{facB}$ through homologous combination at the facB locus was carried out by co-transformation of the $\triangle \mathrm{facB}$ background strain with the $\mathrm{facB}$ (amplified by PCR) open reading frame (ORF, no promoter) and the pyrithiamine-containing plasmid pPTR I at a ratio of $2: 1$. Homologous re-integration of $f a c B$ in the the $\triangle f a c B$ locus was confirmed by PCR and by growth assays (Figure 1B). The facB deletion mutant was constructed using hygromycin as a selectable marker (32) and is therefore resistant to hygromycin and susceptible to pyrithiamine (Figure 1B).

734 Homologous re-integration of $\operatorname{fac} B$ at the $\operatorname{fac} B$ locus will result in the loss of the 
735 hygromycin gene. As pyrithiamine (PT) was used as a marker gene for 736 construction of the re-integration mutant, the resulting strain is resistant to PT 737 (Figure 1B). Growth medium composition was exactly as described previously(40). Radial growth was determined after 5 days whereas dry weight was measured after 3 days of growth. All growth was performed at $37^{\circ} \mathrm{C}$ and experiments were performed in biological triplicates. Reagents were obtained from Sigma unless otherwise specified.

Nuclear magnetic resonance (NMR) analysis. Metabolites were extracted from $5 \mathrm{mg}$ freeze-dried fungal mycelia and dried in a speed vacuum as described previously(28). Extracts were reconstituted in $50 \mu \mathrm{L}$ of deuterated sodium phosphate buffer (100 mM, pH 7.0) containing $0.5 \mathrm{mM}$ TMSP, $3 \mathrm{mM}$ sodium azide and $100 \% \mathrm{D}_{2} \mathrm{O}$. Each sample was sonicated for 10 minutes and vortexed briefly, before a volume of $35 \mu \mathrm{L}$ was transferred into $1.7 \mathrm{~mm} \mathrm{NMR}$ tubes.

Spectra were acquired on a Bruker $600 \mathrm{MHz}$ spectrometer equipped with a TCI 1.7mm z-PFG cryogenic probe and a Bruker SampleJet autosampler. Onedimensional (1D) ${ }^{1} \mathrm{H}$ NMR spectra and $2 \mathrm{D}{ }^{1} \mathrm{H}-{ }^{13} \mathrm{C}$ HSQC spectra were recorded and analysed for each sample as previously described(63).

RNA extraction and cDNA biosynthesis. RNA was extracted with TriZol 754 (Invitrogen) as described previously(40) and $1 \mu \mathrm{g}$ of RNA was reverse 755 transcribed to cDNA using the ImProm II ${ }^{\mathrm{TM}}$ Reverse Transcriptase kit 756 (Promega), according to manufacturer's instructions.

757 RNA-sequencing. The quality of the RNA was assessed using the Agilent 
759 (RIN) value of 7.0. Illumina sequencing was used for sample RNA-sequencing

760 as described previously(64). Libraries were prepared using the

761 TruSeq®Stranded mRNA LT Set B kit (Illumina) and sequenced (2x100bp) on

762 the LNBR NGS sequencing facility HiSeq 2500 instrument. RNA-sequencing

763 data was processed (quality check, clean-up and removal of rRNA and genome

764 mapping) as described previously(64), with the following modifications. The

765 Bioconductor package tximport (version 1.12.3) was used to import raw read

766 counts into DESeq2 (version 1.24.0), which subsequently quantified differential

767 gene expression. Default Benjamini \& Hochberg method was used for multiple

768 hypothesis correction of DESeq2 differentially expressed genes.

769 Enzyme activities. Total cellular proteins were extracted as described 770 previously(65) and isocitrate lyase (ICL) activity was measured and calculated 771 as described previously(65). Acetyl-CoA synthetase (ACS) activity was 772 measured and calculated as described previously(66), with the exception that 773 intracellular proteins were extracted as described above and ACS activity was determined in $50 \mu \mathrm{g}$ total intracellular protein.

High performance liquid chromatography (HPLC) coupled to tandem mass spectrometry (MS/MS) and data analysis. Fungal biomass was separated from supernatant by miracloth before $20 \mathrm{ml}$ of culture supernatants were freezedried. Secondary metabolites (SMs) were extracted from $100 \mathrm{mg}$ freeze-dried sample by re-suspending them in $1 \mathrm{ml} \mathrm{HPLC-grade}$ methanol and sonicating them for $1 \mathrm{~h}$ in an ultrasonic bath. Samples were filtered and dried under a nitrogen stream before being re-suspended in $1 \mathrm{~mL}$ of HPLC-grade methanol. Next, $100 \mu$ of samples were diluted in $900 \mu$ l of methanol and passed through $0.22 \mu \mathrm{m}$ filters into vials. 
784

785

786

787

788

789

790

791

792

793

794

795

796

797

798

799

800

801

802

803

804

805

806

807

808

HPLC MS/MS analysis was performed using a Thermo Scientific QExactive ${ }^{\circledR}$ Hybrid Quadrupole-Orbitrap Mass Spectrometer. Parameters were as follows: positive mode, $+3.5 \mathrm{kV}$ capillary voltage; $250{ }^{\circ} \mathrm{C}$ capillary temperature; $50 \mathrm{~V} \mathrm{~S}$ lens and a $\mathrm{m} / \mathrm{z}$ range of $133.40-2000.00$. MS/MS was performed using a normalized collision energy (NCE) of $30 \mathrm{eV}$ and 5 precursors per cycle were selected. For the stationary phase the Thermo Scientific Accucore C18 $2.6 \mu \mathrm{m}$ (2.1 $\mathrm{mm} \times 100 \mathrm{~mm}$ ) column was used. The mobile phase was carried out using $0.1 \%$ formic acid $(\mathrm{A})$ and acetonitrile $(\mathrm{B})$ and the following gradient was applied: 0-10 $\min 5 \%$ B up to $98 \%$ B; hold for 5 min; $15-16.295 \%$ B up to $5 \%$ B; hold for $8.8 \mathrm{~min}$. The total run time was $25 \mathrm{~min}$ and the flow rate was $0.2 \mathrm{~mL} \mathrm{~min}^{-1}$ with 3 $\mu \mathrm{L}$ injection volume. Data analysis was conducted using the Xcalibur software, version 3.0.63 (Thermo Fisher Scientific).

Molecular networks were made using the Global Natural Products Social Molecular Networking (GNPS) website (https://ccmsucsd.github.io/GNPSDocumentation/ from http:/gnps.ucsd.edu). First, all MS/MS fragment ions within +/- $17 \mathrm{Da}$ of the precursor $\mathrm{m} / \mathrm{z}$ were removed and spectra were filtered by choosing only the top 6 fragment ions in the +/- $50 \mathrm{Da}$ window for the entire spectrum. The precursor ion mass tolerance and the MS/MS fragment ion tolerance were set to 0.02 Da. Subsequently, networks were created where edges were filtered to have a cosine score higher than 0.6 and more than 5 matched peaks. Edges between two nodes were kept in the network only if each of the nodes appeared in each other's respective top 10 most similar nodes. Finally, the maximum size of a molecular family was set to 100 , and the lowest scoring edges were removed. Network spectra were then searched against the GNPS spectral libraries and library spectra were filtered in 
809 the same manner as the input data. Matches between network spectra and

810 library spectra were filtered to have a score higher than 0.6 and at least 5 811 matching peaks (67). GNPS data used in this work are available at:

812 https://gnps.ucsd.edu/ProteoSAFe/status.jsp?task=f815e5618b05433fb768299 813 a351fb793 (72 h data).

814 Cell wall polysaccharide quantification. Strains were grown for $24 \mathrm{~h}$ from $1 \mathrm{x}$ $81510^{8}$ conidia in $50 \mathrm{ml}$ minimal medium (MM) supplemented with $1 \%(\mathrm{w} / \mathrm{v})$ glucose or sodium acetate. Mycelia were harvested by vacuum filtration, washed, re817 suspended in $30 \mathrm{ml}$ of $\mathrm{ddH}_{2} \mathrm{O}$ and disrupted using $5 \mathrm{ml}$ of $0.5 \mathrm{~mm}$ glass beads 818 in the FastPrep (MP Biomedicals) homogenizer at $4^{\circ} \mathrm{C}$ with two cycles of $60 \mathrm{~s}$ 819 (6.0 vibration unit) and a 5 min interval between both cycles. Samples were centrifuged at $5000 \mathrm{rpm}$ for $10 \mathrm{~min}$ at $4^{\circ} \mathrm{C}$, before the cell wall-containing pellets were washed 3 times with $\mathrm{ddH}_{2} \mathrm{O}$, re-suspended in $15 \mathrm{ml}$ of $50 \mathrm{mM} \mathrm{pH} 7.5$ Tris$\mathrm{HCl}, 50 \mathrm{mM}$ EDTA, 2\% w/v SDS (2\%) and $40 \mathrm{mM} \beta$-Mercaptoethanol and boiled twice for $15 \mathrm{~min}$ in a water-bath. Samples were centrifuged at $5000 \mathrm{rpm}$ for 10 min and washed 5 times with $\mathrm{ddH}_{2} \mathrm{O}$. Resultant cell wall fractions were freeze-dried and the dry-weight was measured. Alkali-fractionation of the cell wall was carried out by incubating them twice in $1 \mathrm{M} \mathrm{NaOH}$ containing $0.5 \mathrm{M}$ $\mathrm{NaBH}_{4}$ at $70^{\circ} \mathrm{C}$ for $1 \mathrm{~h}$. Samples were centrifuged to separate supernatant [alkali-soluble (AS) fraction] from the pellet [alkali-insoluble (AI) fraction]. The $\mathrm{Al}$ fractions was washed six times with $\mathrm{ddH}_{2} \mathrm{O}$ and centrifuged at $5000 \mathrm{rpm}$ for 10 min and freeze-dried. The excess of $\mathrm{NaBH}_{4}$ in the alkali-soluble fraction (AS) was neutralized with $2 \% \mathrm{v} / \mathrm{v}$ acetic acid, dialyzed against water until they achieved a neutral $\mathrm{pH}$ and freeze-dried. Subsequently, Al and AS fractions were subjected to gas liquid chromatography as previously described(68). 
834 Minimal inhibitory concentrations (MICs). MICs of amphotericin B and azoles

835 on the $A$. fumigatus wild-type (WT) strain were carried out as described 836 previously(69) with the exception that the WT strain was also grown in MM supplemented with glucose (GMM) or acetate (AMM).

Neutrophil-mediated killing of hyphae. Assessing the viability of $A$. fumigatus hyphae in the presence of human neutrophils was carried out as described previously with modifications(70). Briefly, human polymorphonuclear cells (PMNs) were isolated from $8 \mathrm{~mL}$ of peripheral blood of adult male healthy volunteers by density centrifugation and re-suspended in Hank's Balanced Salt Solution (Gibco®). $1 \times 10^{8}$ A. fumigatus conidia were incubated for $8 \mathrm{~h}$ or $13 \mathrm{~h}$ at $37^{\circ} \mathrm{C}$ in $30 \mathrm{ml}$ GMM or AMM on a rotary shaker before they were centrifuged for $5 \mathrm{~min}$ at $4000 \mathrm{rpm}$, supernatants were discarded and pellets were resuspended in $1 \mathrm{ml}$ PBS (phosphate buffered saline). To assess the percentage of germinated conidia, samples were viewed under a microscope (Zeiss) at 100x magnification before a total of 100 conidia were counted and the \% of germinated conidia was calculated. Pre-grown hyphae were then incubated with neutrophils $\left(0,1,2\right.$ or $3 \times 10^{5}$ cells $\left./ \mathrm{ml}\right)$ for $1 \mathrm{~h}$ at $37^{\circ} \mathrm{C}$ in RPMI medium before cells were lysed and the MTT [3-(4,5-dimethylthiazol-2-yl)-2,5diphenyltetrazolium bromide] assay was performed. Hyphal viability was calculated as a percentage of its viability after incubation without neutrophils.

Bone marrow-derived macrophage (BMDM) phagocytosis and killing assays. BMDM preparation and the ability to kill $A$. fumigatus conidia, as determined by assessing colony forming units (CFU), was carried out exactly as

857 described previously(71). The ability of BMDMs to phagocytise A. fumigatus conidia was carried out exactly as described in(72). Fresh $A$. fumigatus conidia 
859 were harvested from plates in PBS and filtered through Miracloth (Calbiochem).

860 Conidial suspensions were washed three times with PBS and counted using a 861 hemocytometer. For the killing assay, a dilution of $1 \times 10^{5}$ conidia in $200 \mu$ RPMIFCS was prepared. For the phagocytosis assay, $1 \times 10^{6}$ conidia were resuspended in $1 \mathrm{ml}$ PBS and inactivated under UV light for $2 \mathrm{~h}$. The percentage of phagocytised conidia was calculated based on conidia cell wall staining with calcofluor white (CFW) (phagocytised conidia are not stained).

Infection of Galleria mellonella. Breeding and selection of wax moth larvae, preparation of $A$. fumigatus conidia and infection of the last left proleg of larvae with $A$. fumigatus was carried out exactly as described previously(73).

Ethics statement. Eight-week-old gender- and age-matched C57BL/6 mice were bred under the specific-pathogen-free condition and kept at the Life and

871 Health Sciences Research Institute (ICVS) Animal Facility. Animal 872 experimentation was performed following biosafety level 2 (BSL-2) protocols approved by the Institutional Animal Care and Use Committee (IACUC) of

874 University of Minho, and the ethical and regulatory approvals were consented by the Ethics Subcommittee for Life and Health Sciences (no. 074/016). All procedures followed the EU-adopted regulations (Directive 2010/63/EU) and were conducted according to the guidelines sanctioned by the Portuguese ethics committee for animal experimentation, Direção-Geral de Alimentação e Veterinária (DGAV).

Infection of chemotherapeutic mice, fungal burden and histopathology.

Mice were immunosuppressed intraperitoneally (i.p.) with $200 \mathrm{mg} / \mathrm{Kg}$ of cyclophosphamide (Sigma) on days $-4,-1$, and +2 prior to and post infection, 
883

884

885

886

887

888

889

890

891

892

893

894

895

896

897

898

899

900

901

902

903

904

905

906

and subcutaneously with $150 \mathrm{mg} / \mathrm{Kg}$ hydrocortisone acetate (Acros Organics) on day -1 prior to infection. A. fumigatus conidia suspensions were prepared freshly a day prior to infection and washed three times with PBS. The viability of the administered conidia was determined by growing them in serial dilutions on complete (YAG) medium at $37^{\circ} \mathrm{C}$. Mice $(n=10 /$ strain) were infected by intranasal instillation of $1 \times 10^{6}$ conidia in $20 \mu$ of PBS. Mice $(n=5)$ which received $20 \mu \mathrm{l}$ of PBS were used as negative control. To avoid bacterial infections, the animals were treated with $50 \mu \mathrm{g} / \mathrm{mL}$ of chloramphenicol in drinking water ad libitum. Animals were weighed daily and sacrificed in case of $20 \%$ loss weight, severe ataxia or hypothermia, and other severe complications.

For histological analysis, the lungs were perfused with PBS, excised, and fixed with $10 \%$ buffered formalin solution for at least 48 hours, and paraffin embedded. Lung sections were stained with hematoxylin and eosin (H\&E) for pathological examination.Paraffin-embedded lung tissue sections were also stained for the presence of fungal structures using the Silver Stain Kit (SigmaAldrich), according to the manufacturer's instructions. Images were acquired using a BX61 microscope (Olympus) and a DP70 high-resolution camera (Olympus). To quantify lung inflammation of infected animals, inflamed areas on slide images were analysed using the thresholding tool in ImageJ software (v1.50i, NIH, USA) according to the manufacturer's instructions.

Data Availability. The RNAseq dataset can be accessed at NCBl's Short Read Archive under the Bioproject ID: PRJNA668271. 
1. Denning DW, Bromley MJ. 2015. How to bolster the antifungal pipeline. Science (80-. ). 347:1414-1416.

2. Latgé J-P, Chamilos G. 2020. Aspergillus fumigatus and Aspergillosis in 2019. Clin. Microbiol. Rev. 33:1-75.

3. Ramachandra S, Linde J, Brock M, Guthke R, Hube B, Brunke S. 2014. Regulatory networks controlling nitrogen sensing and uptake in

4. Matthaiou El, Sass G, Stevens DA, Hsu JL. 2018. Iron: an essential nutrient for Aspergillus fumigatus and a fulcrum for pathogenesis. Curr. Opin. Infect. Dis. 31:506-511.

5. Vicentefranqueira R, Leal F, Marín L, Sánchez CI, Calera JA. 2019. The interplay between zinc and iron homeostasis in Aspergillus fumigatus under zinc-replete conditions relies on the iron-mediated regulation of alternative transcription units of zafA and the basal amount of the ZafA zinc-responsiveness transcription fac. Environ. Microbiol. 21:2787-2808.

925 7. Ries LNA, Beattie S, Cramer RA, Goldman GH. 2018. Overview of carbon and nitrogen catabolite metabolism in the virulence of human pathogenic fungi. Mol. Microbiol. 107.

8. Grahl N, Puttikamonkul S, Macdonald JM, Gamcsik MP, Ngo LY,

Hohl TM, Cramer RA. 2011. In vivo hypoxia and a fungal alcohol 
dehydrogenase influence the pathogenesis of invasive pulmonary aspergillosis. PLoS Pathog. 7.

9. Beattie SR, Mark KMK, Thammahong A, Ries LNA, Dhingra S, Caffrey-Carr AK, Cheng C, Black CC, Bowyer P, Bromley MJ, Obar JJ, Goldman GH, Cramer RA. 2017. Filamentous fungal carbon catabolite repression supports metabolic plasticity and stress responses essential for disease progression. PLoS Pathog. 13.

10. Schug ZT, Vande Voorde J, Gottlieb E. 2016. The metabolic fate of acetate in cancer. Nat. Rev. Cancer 16:708-717.

11. Li M, van Esch BCAM, Wagenaar GTM, Garssen J, Folkerts G, Henricks PAJ. 2018. Pro- and anti-inflammatory effects of short chain fatty acids on immune and endothelial cells. Eur. J. Pharmacol. 831:5259.

12. Dickson RP, Erb-Downward JR, Martinez FJ, Huffnagle GB. 2016. The Microbiome and the Respiratory Tract. Annu Rev Physiol 10:481504.

13. Anand S, Mande SS. 2018. Diet, microbiota and gut-lung connection. Front. Microbiol. 9.

14. Sugui JA, Kim HS, Zarember KA, Chang YC, Gallin JI, Nierman WC, Kwon-Chung KJ. 2008. Genes differentially expressed in conidia and hyphae of Aspergillus fumigatus upon exposure to human neutrophils. PLoS One 3.

15. Barelle CJ, Priest CL, MacCallum DM, Gow NAR, Odds FC, Brown 
AJP. 2006. Niche-specific regulation of central metabolic pathways in a fungal pathogen. Cell. Microbiol. 8:961-971.

16. Chen Y, Toffaletti DL, Tenor JL, Litvintseva AP, Fang C, Mitchell TG, McDonald TR, Nielsen K, Boulware DR, Bicanic T, Perfect JR. 2014. The Cryptococcus neoformans Transcriptome at the Site of Human Meningitis. MBio 5:277-297.

17. McDonagh A, Fedorova ND, Crabtree J, Yu Y, Kim S, Chen D, Loss O, Cairns T, Goldman G, Armstrong-James D, Haynes K, Haas H, Schrettl M, May G, Nierman WC, Bignell E. 2008. Sub-telomere directed gene expression during initiation of invasive aspergillosis. PLoS Pathog. 4.

18. Bertuzzi M, Schrettl M, Alcazar-Fuoli L, Cairns TC, Muñoz A, Walker LA, Herbst S, Safari M, Cheverton AM, Chen D, Liu H, Saijo S, Fedorova ND, Armstrong-James D, Munro CA, Read ND, Filler SG, Espeso EA, Nierman WC, Haas H, Bignell EM. 2014. The pHResponsive PacC Transcription Factor of Aspergillus fumigatus Governs Epithelial Entry and Tissue Invasion during Pulmonary Aspergillosis. PLoS Pathog. 10.

19. Kale SD, Ayubi T, Chung D, Tubau-Juni N, Leber A, Dang HX, Karyala S, Hontecillas R, Lawrence CB, Cramer RA, BassaganyaRiera J. 2017. Modulation of Immune Signaling and Metabolism Highlights Host and Fungal Transcriptional Responses in Mouse Models of Invasive Pulmonary Aspergillosis. Sci. Rep. 7:1-25.

20. Liu H, Xu W, Bruno VM, Phan QT, Solis N V., Woolford CA, Ehrlich 
RL, Shetty AC, McCraken C, Lin J, Bromley MJ, Mitchell AP, Filler SG. 2021. Determining Aspergillus fumigatus transcription factor expression and function during invasion of the mammalian lung. PLoS Pathog. 17.

21. Albrecht D, Guthke R, Brakhage AA, Kniemeyer O. 2010. Integrative analysis of the heat shock response in Aspergillus fumigatus. BMC Genomics 11.

22. Vödisch M, Scherlach K, Winkler R, Hertweck C, Braun HP, Roth M, Haas H, Werner ER, Brakhage AA, Kniemeyer O. 2011. Analysis of the Aspergillus fumigatus proteome reveals metabolic changes and the activation of the pseurotin A biosynthesis gene cluster in response to hypoxia. J. Proteome Res. 10:2508-2524.

23. Sá-Pessoa J, Amillis S, Casal M, Diallinas G. 2015. Expression and specificity profile of the major acetate transporter AcpA in Aspergillus nidulans. Fungal Genet. Biol. 76:93-103.

24. Hynes MJ, Murray SL, Andrianopoulos A, Davis MA. 2011. Role of carnitine acetyltransferases in acetyl coenzyme a metabolism in Aspergillus nidulans. Eukaryot. Cell 10:547-555.

25. Todd RB, Andrianopoulos A, Davis MA, Hynes MJ. 1998. FacB, the Aspergillus nidulans activator of acetate utilization genes, binds dissimilar DNA sequences. EMBO J. 17:2042-2054.

26. Hynes MJ, Murray SL, Duncan A, Khew GS, Davis MA. 2006. Regulatory genes controlling fatty acid catabolism and peroxisomal functions in the filamentous fungus Aspergillus nidulans. Eukaryot. Cell 
5:794-805.

1002

1003

1004

1005

1006

1007

1008

1009

1010

1011

1012

1013

1014

1015

1016

1017

1018

1019

1020

1021

1022

1023

1024

27. McCammon MT. 1996. Mutants of Saccharomyces cerevisiae with defects in acetate metabolism: Isolation and characterization of Acnmutants. Genetics 144:57-69.

28. Ries LNA, de Assis LJ, Rodrigues FJS, Caldana C, Rocha MC, Malavazi I, Bayram Ö, Goldman GH. 2018. The Aspergillus nidulans pyruvate dehydrogenase kinases are essential to integrate carbon source metabolism. G3 Genes, Genomes, Genet. 8.

29. Rodrigues ML, Rozental S, Couceiro JNSS, Angluster J, Alviano CS, Travassos LR. 1997. Identification of N-Acetylneuraminic acid and its 9O-acetylated derivative on the cell surface of Cryptococcus neoformans. Influence on fungal phagocytosis. Infect. Immun. 65:4937-4942.

30. Soares R, de A Soares R, Alviano D, Angluster J, Alviano C, Travassos L. 2000. Identification of sialic acids on the cell surface of Candida albicans. Biochim Biophys Acta 1474:262-268.

31. Warwas ML, Watson JN, Bennet AJ, Moore MM. 2007. Structure and role of sialic acids on the surface of Aspergillus fumigatus conidiospores. Glycobiology 17:401-410.

32. Furukawa T, van Rhijn N, Fraczek M, Gsaller F, Davies E, Carr P, Gago S, Fortune-Grant R, Rahman S, Gilsenan JM, Houlder E, Kowalski CH, Raj S, Paul S, Cook P, Parker JE, Kelly S, Cramer RA, Latgé JP, Moye-Rowley S, Bignell E, Bowyer P, Bromley MJ. 2020. The negative cofactor 2 complex is a key regulator of drug resistance in Aspergillus fumigatus. Nat. Commun. 11. 
33. Pongpom M, Liu H, Xu W, Snarr BD, Sheppard DC, Mitchell AP, Filler SG. 2015. Divergent targets of Aspergillus fumigatus AcuK and AcuM transcription factors during growth in vitro versus invasive disease. Infect. Immun. 83:923-933.

34. Smith TD, Calvo AM. 2014. The mtfA transcription factor gene controls morphogenesis, gliotoxin production, and virulence in the opportunistic human pathogen Aspergillus fumigatus. Eukaryot. Cell 13:766-775.

35. de Assis LJ, Ries LNA, Savoldi M, Dinamarco TM, Goldman GH, Brown NA. 2015. Multiple phosphatases regulate carbon sourcedependent germination and primary metabolism in aspergillus nidulans. G3 Genes, Genomes, Genet. 5.

36. Adnan M, Zheng W, Islam W, Arif M, Abubakar YS, Wang Z, Lu G. 2018. Carbon catabolite repression in filamentous Fungi. Int. J. Mol. Sci. 19:1-23.

37. Candida Y, Sandai D, Zhikang Y, Selway L. 2012. Carbon Assimilation in the Pathogenic The Evolutionary Rewiring of Ubiquitination Targets Has 3:1-13.

38. Childers DS, Raziunaite I, Mol Avelar G, Mackie J, Budge S, Stead D, Gow NAR, Lenardon MD, Ballou ER, MacCallum DM, Brown AJP. 2016. The Rewiring of Ubiquitination Targets in a Pathogenic Yeast Promotes Metabolic Flexibility, Host Colonization and Virulence. PLoS Pathog. 12:1-26.

39. Reis TF Dos, Menino JF, Bom VLP, Brown NA, Colabardini AC, Savoldi M, Goldman MHS, Rodrigues F, Goldman GH. 2013. 
Identification of glucose transporters in aspergillus nidulans. PLoS One 8.

1050

1051

1052

1053

1054

1055

1056

1057

1058

1059

1060

1061

1062

1063

1064

1065

1066

1067

1068

1069

1070

1071

1072

40. Ries LNA, Beattie SR, Espeso EA, Cramer RA, Goldman GH. 2016. Diverse regulation of the CreA carbon catabolite repressor in aspergillus nidulans. Genetics 203.

41. Raffa N, Keller NP. 2019. A call to arms: Mustering secondary metabolites for success and survival of an opportunistic pathogen. PLoS Pathog. 15:1-9.

42. Bignell E, Cairns TC, Throckmorton K, Nierman WC, Keller NP. 2016. Secondary metabolite arsenal of an opportunistic pathogenic fungus. Philos. Trans. R. Soc. B Biol. Sci. 371:1-9.

43. Tabata N, Tanaka H, Omura S. 1996. Pyripyropenes, Novel ACATInhibitors Produced by Aspergillus fumigatus IV . Structure Elucidation of Pyripyropenes Mto R Wehave reported pyripyropenes A to $\mathrm{L}$, a novel series of polyoxygenated metabolites produced by Aspergillus 292-298.

44. Clavaud C, Beauvais A, Barbin L, Munier-Lehmann H, Latgé JP. 2012. The composition of the culture medium influences the $\beta-1,3-$ glucanmetabolism of Aspergillus fumigatus and the antifungal activity of inhibitors of $\beta-1,3$-glucan synthesis. Antimicrob. Agents Chemother. 56:3428-3431.

45. de Assis LJ, Manfiolli A, Mattos E, Jacobsen ID, Brock M, Cramer RA, Thammahong A, Hagiwara D, Nicolas L, Ries A, Goldman GH. 2018. Protein Kinase A and High-Osmolarity Glycerol Response Pathways Cooperatively Control Cell Wall Carbohydrate Mobilization in 
Aspergillus fumigatus. MBio 9.

46. Latgé J-P, Beauvais A, Chamilos G. 2017. The Cell Wall of the Human Fungal Pathogen Aspergillus fumigatus: Biosynthesis, Organization, Immune Response, and Virulence . Annu. Rev. Microbiol. 71:99-116.

47. Hiemenz JW, Raad II, Maertens JA, Hachem RY, Saah AJ, Sable CA, Chodakewitz JA, Severino ME, Saddier P, Berman RS, Ryan DM, Dinubile MJ, Patterson TF, Denning DW, Walsh TJ. 2010. Efficacy of caspofungin as salvage therapy for invasive aspergillosis compared to standard therapy in a historical cohort. Eur. J. Clin. Microbiol. Infect. Dis. 29:1387-1394.

48. Loiko V, Wagener J. 2017. The paradoxical effect of echinocandins in Aspergillus fumigatus relies on recovery of the $\beta-1,3$-glucan synthase Fks1. Antimicrob. Agents Chemother. 61:1-11.

49. Ries LNA, Steenwyk JL, De Castro PA, De Lima PBA, Almeida F, De Assis LJ, Manfiolli AO, Takahashi-Nakaguchi A, Kusuya Y, Hagiwara D, Takahashi H, Wang X, Obar JJ, Rokas A, Goldman GH. 2019. Nutritional heterogeneity among aspergillus fumigatus strains has consequences for virulence in a strain- And host-dependent manner. Front. Microbiol. 10:1-20.

50. Katz ME, Hynes MJ. 1989. Isolation and analysis of the acetate regulatory gene, facB, from Aspergillus nidulans. Mol. Cell. Biol. 9:56965701.

51. Stemple CJ, Davis MA, Hynes MJ. 1998. The facC gene of Aspergillus nidulans encodes an acetate-inducible carnitine acetyltransferase. J. 
Bacteriol. 180:6242-6251.

1098

1099

52. Liu Y, Beyer A, Aebersold R. 2016. On the Dependency of Cellular Protein Levels on mRNA Abundance. Cell 165:535-550.

53. de Assis LJ, Ulas M, Ries LNA, EI Ramli NAM, Sarikaya-Bayram O, Braus GH, Bayram O, Goldman GH. 2018. Regulation of Aspergillus nidulans CreA-mediated catabolite repression by the F-Box proteins Fbx23 and Fbx47. MBio 9.

54. Portnoy T, Margeot A, Linke R, Atanasova L, Fekete E, Sándor E, Hartl L, Karaffa L, Druzhinina IS, Seiboth B, Le Crom S, Kubicek CP. 2011. The CRE1 carbon catabolite repressor of the fungus Trichoderma reesei: A master regulator of carbon assimilation. BMC Genomics 12:269.

55. Keller NP. 2019. Fungal secondary metabolism: regulation, function and drug discovery. Nat. Rev. Microbiol. 17:167-180.

56. Tortorano AM, Dannaoui E, Meletiadis J, Mallie M, Viviani MA, Piens MA, Rigoni AL, Bastide JM, Grillot R, Lebeau B, Burnod J, Nolard N, Symoens F, Goens K, Heinermann S, Bertout S, Castel D, Renaud F, De Mëeus T, Perraud M, Monier MF, Chapuis F, Cogliati M, Barton R, Evans EGV, Ashbee HR, Hopwood V, Meis JF, Voss A, Verweij PE, Donnelly JP, Rath PM, Ansorg R. 2002. Effect of medium composition on static and cidal activity of amphotericin B, itraconazole, voriconazole, posaconazole and terbinafine against Aspergillus fumigatus: A multicenter study. J. Chemother. 14:246-252.

57. GHANNOUM MAHMOUD A., RICE LOUIS B. 1999. Antifungal Agents: Mode of Action, Mechanisms of Resistance, and Correlation of These 
Mechanisms with Bacterial Resistance. Clin. Microbiol. Rev. 12:501-517.

58. Shepardsona KM, Ngoc LY, Aimaniandad V, Latge J-P, Barkera BM, Blossera SJ, Iwakurae Y, Hohl TM, Cramer RA. 2013. Hypoxia enhances innate immune activation to Aspergillus fumigates through cell wall modulation. Microbes Infect. 15:259-269.

59. Viola A, Munari F, Sánchez-Rodríguez R, Scolaro T, Castegna A. 2019. The metabolic signature of macrophage responses. Front. Immunol. 10:1-16.

60. Zhuge X, Sun Y, Jiang M, Wang J, Tang F, Xue F, Ren J, Zhu W, Dai J. 2019. Acetate metabolic requirement of avian pathogenic Escherichia coli promotes its intracellular proliferation within macrophage. Vet. Res. $50: 31$.

61. Schöbel F, Ibrahim-Granet O, Avé P, Latgé JP, Brakhage AA, Brock M. 2007. Aspergillus fumigatus does not require fatty acid metabolism via isocitrate lyase for development of invasive aspergillosis. Infect. Immun. $75: 1237-1244$.

62. Olivas I, Royuela M, Romero B, Monteiro MC, Mínguez JM, Laborda F, Lucas JR De. 2008. Ability to grow on lipids accounts for the fully virulent phenotype in neutropenic mice of Aspergillus fumigatus null mutants in the key glyoxylate cycle enzymes. Fungal Genet. Biol. 45:4560.

63. Saborano R, Eraslan Z, Roberts J, Khanim FL, Lalor PF, Reed MAC, Günther UL. 2019. A framework for tracer-based metabolism in mammalian cells by NMR. Sci. Rep. 9:1-13. 
1145 64. Pereira Silva L, Alves de Castro P, dos Reis TF, Paziani MH, Von

1146

1147

1148

Zeska Kress MR, Riaño-Pachón DM, Hagiwara D, Ries LNA, Brown NA, Goldman GH. 2017. Genome-wide transcriptome analysis of Aspergillus fumigatus exposed to osmotic stress reveals regulators of osmotic and cell wall stresses that are SakA ${ }^{\mathrm{HOG} 1}$ and $\mathrm{MpkC}$ dependent. Cell. Microbiol. 19.

65. Ries LNA, José de Assis L, Rodrigues FJS, Caldana C, Rocha MC, Malavazi I, Bayram Ö, Goldman GH. 2018. The Aspergillus nidulans Pyruvate Dehydrogenase Kinases Are Essential To Integrate Carbon Source Metabolism . G3\&amp;\#58; Genes|Genomes|Genetics 8:24452463.

66. Castano-Cerezo S, Bernal V, Canovas M. 2012. http://www.bioprotocol.org/e256 Vol 2, Iss 17, Sep 05, 2012. Bio-protocol 2:e256.

67. Wang M, Carver JJ, Phelan V V., Sanchez LM., Garg N., Peng Y., Nguyen DD., Watrous J., Kapono CA., Luzzatto-Knaan T., Porto C., Bouslimani A., Melnik AV., Meehan MJ., Liu W-T., Crüsemann M., Boudreau PD., Esquenazi E., Sandoval-Calderon M., Kersten RD., Pace LA., Quinn RA., Duncan KR., Hsu C-C., Floros DJ., Gavilan RG., Kleigrewe K., Northen T., Dutton RJ., Parrot D., Carlson EE., Aigle B., Michelsen CF., Jelsbak L., Sohlenkamp C., Pevzner P., Edlund A., McLean J., Piel J., Murphy BT., Gerwick L., Liaw C-C., Yang Y-L., Humpf H-U., Maansson M., Keyzers RA., Sims AC., Johnson AR., Sidebottom AM., Sedio BE., Klitgaard A., Larson CB., Boya P CA., TorresMendoza D., Gonzalez DJ., Silva DB., Marques LM., Demarque 
DP., Pociute E., O'Neill EC., Briand E., Helfrich EJN., Granatosky EA., Glukhov E., Ryffel F., Houson H., Mohimani H., Kharbush JJ., Zeng Y., Vorholt JA., Kurita KL., Charusanti P., McPhail KL., Nielsen KF., Vuong L., Elfeki M., Traxler MF., Engene N., Koyama N., Vining OB., Baric R., Silva RR., Mascuch SJ., Tomasi S., Jenkins S., Macherla V., Hoffman T., Agarwal V., Williams PG., Dai J., Neupane R., Gurr J., Rodríguez AMC., Lamsa A., Zhang C., Dorrestein K., Duggan BM., Almaliti J., Allard P-M., Phapale P., Nothias L-F., Alexandrov T., Litaudon M., Wolfender J-L., Kyle JE., Metz TO., Peryea T., Nguyen D-T., VanLeer D., Shinn P., Jadhav A., Müller R., Waters KM., Shi W., Liu X., Zhang L., Knight R., Jensen PR., Palsson BØ., Pogliano K., Linington RG., Gutierrez M., Lopes NP.', Gerwick WH., Moore BS., Dorrestein PC., Bandeira N. 2017. Sharing and community curation of mass spectrometry data with GNPS. Nat. Biotechnol. 34:828-837.

68. Richie DL, Hartl L, Aimanianda V, Winters MS, Fuller KK, Miley MD, White S, McCarthy JW, Latgé JP, Feldmesser M, Rhodes JC, Askew DS. 2009. A role for the unfolded protein response (UPR) in virulence and antifungal susceptibility in Aspergillus fumigatus. PLoS Pathog. 5.

69. Bastos RW, Valero C, Silva LP, Schoen T, Drott M, Brauer V, SilvaRocha R, Lind A, Steenwyk JL, Rokas A, Rodrigues F, ResendizSharpe A, Lagrou K, Marcet-Houben M, Gabaldón T, McDonnell E, Reid I, Tsang A, Oakley BR, Loures FV, Almeida F, Huttenlocher A, Keller NP, Ries LNA, Goldmana GH. 2020. Functional characterization of clinical isolates of the opportunistic fungal pathogen Aspergillus nidulans. bioRxiv 2020.01.28.917278. 
70. Steenwyk JL, Lind AL, Ries LNA, dos Reis TF, Silva LP, Almeida F, Bastos RW, Fraga da Silva TF de C, Bonato VLD, Pessoni AM, Rodrigues F, Raja HA, Knowles SL, Oberlies NH, Lagrou K, Goldman GH, Rokas A. 2020. Pathogenic Allodiploid Hybrids of Aspergillus Fungi. Curr. Biol. 30:2495-2507.e7.

71. de Castro PA, Colabardini AC, Manfiolli AO, Chiaratto J, Silva LP, Mattos EC, Palmisano G, Almeida F, Persinoti GF, Ries LNA, Mellado L, Rocha MC, Bromley M, Silva RN, de Souza GS, Loures FV, Malavazi I, Brown NA, Goldman GH. 2019. Aspergillus fumigatus calcium-responsive transcription factors regulate cell wall architecture promoting stress tolerance, virulence and caspofungin resistancePLoS Genetics.

72. Rocha MC, de Godoy KF, Bannitz-Fernandes R, Fabri JHTM, Barbosa MMF, de Castro PA, Almeida F, Goldman GH, da Cunha AF, Netto LES, de Oliveira MA, Malavazi I. 2018. Analyses of the three 1Cys Peroxiredoxins from Aspergillus fumigatus reveal that cytosolic Prx1 is central to $\mathrm{H} 2 \mathrm{O} 2$ metabolism and virulence. Sci. Rep. 8:1-18.

73. dos Reis TF, Silva LP, de Castro PA, de Lima PBA, do Carmo RA, Marini MM, da Silveira JF, Ferreira BH, Rodrigues F, Malavazi I, Goldman GH. 2018. The influence of genetic stability on Aspergillus fumigatus virulence and azole resistance. G3 Genes, Genomes, Genet. 8:265-278. 
1218

1219

1220

1221

1222

1223

1224

1225

1226

1227

1228

1229

1230

1231

1232

1233

1234

1235

1236

1237

1238

1239

1240

1241

1242

\section{Acknowledgements}

We are grateful to the Henry Welcome Building for Biomolecular NMR staff at the University of Birmingham for supporting access to NMR instruments. We would also like to thank the Brazilian Biorenewables National Laboratory (LNBR) for using the NGS sequencing facility to generate the RNA-seq data.

We would like to thank the São Paulo Research Foundation (FAPESPFundacao de Amparo a Pesquisa do Estado de Sao Paulo, Brazil) grant numbers 2017/14159-2 (LNAR), 2016/12948-7 (PAC), 2017/08750-0 (TFR), 2016/07870-9 (GHG), 2018/00715-3 (CV) and the Conselho Nacional de Desenvolvimento Científico e Tecnológico, Brazil (CNPq) grant numbers 301058/2019-9 and 404735/2018-5 (GHG) for financial support. IFD acknowledges CICECO-Aveiro Institute of Materials (UIDB/50011/2020 \& UIDP/50011/2020) financed by national funds through the Foundation for Science and Technology/MCTES, and the National NMR Network (PTNMR) partially supported by Infrastructure Project № 022161 (co-financed by FEDER through COMPETE 2020, POCI and PORL and FCT through PIDDAC). AC, RAG, CDO were supported by the Fundação para a Ciência e a Tecnologia (FCT) (PTDC/MED-GEN/28778/2017, UIDB/50026/2020 and UIDP/50026/2020). Additional support was provided by the Northern Portugal Regional Operational Programme (NORTE 2020), under the Portugal 2020 Partnership Agreement, through the European Regional Development Fund (ERDF) (NORTE-01-0145-FEDER-000013 and NORTE-01-0145-FEDER000023), the European Union's Horizon 2020 research and innovation programme under grant agreement no. 847507, and the "la Caixa" Foundation (ID 100010434) and FCT under the agreement LCF/PR/HP17/52190003. 
1243 Individual support was provided by FCT (SFRH/BD/141127/2018 to CDO, and 1244 CEECIND/03628/2017 to AC). This study was financed in part by the 1245 Coordenação de Aperfeiçoamento de Pessoal de Nível Superior - Brasil (CAPES) - Finance Code 001 (JHC scholarship). SSWW was supported by

Pasteur-Roux-Cantarini fellowship. JLS and AR are supported by the Howard Hughes Medical Institute through the James H. Gilliam Fellowships for Advanced Study program; AR is additionally supported by the National Institutes of Health / National Institute of Allergy and Infectious Diseases (1R56Al146096-01A1).

\section{Figure legends}

Figure 1. Acetate metabolism in A. fumigatus. A. One- and two-dimensional $(1 \mathrm{D}, 2 \mathrm{D}) \mathrm{NMR}$ (nuclear magnetic resonance) analysis of ${ }^{13} \mathrm{C}_{2}$-labelled acetate incorporation and metabolism: (top) Expansion of $1 \mathrm{D}^{1} \mathrm{H}$ NMR spectra of fungal cell extracts showing the increase in acetate carbon satellite peaks upon culture of $A$. fumigatus in ${ }^{13} \mathrm{C}_{2}$-acetate-containing medium; (bottom) ${ }^{13} \mathrm{C} /{ }^{12} \mathrm{C}$ ratios for metabolites that incorporated ${ }^{13} \mathrm{C}$-derived from acetate, as determined through integration of ${ }^{1} \mathrm{H}^{-13} \mathrm{C} \mathrm{HSQC}$ (heteronuclear single quantum coherence) spectra recorded for extracts of fungal cells grown for 5 and $15 \mathrm{~min}$ in medium containing ${ }^{13} \mathrm{C}_{2}$-acetate in comparison to non-labelled control cultures. Standard deviations represent the average of biological triplicates. B-D. The transcription factor FacB is essential for growth in the presence of acetate. Strains were grown for 5 days $(B, C)$ or 3 days $(D)$ in either solid $(B, C)$ or liquid $(D)$ minimal medium supplemented with $1 \% \mathrm{w} / \mathrm{v}$ glucose (gluc, B, D), $0.5 \% \mathrm{w} / \mathrm{v}(\mathrm{B})$ or $1 \%$ 
$1267 \mathrm{w} / \mathrm{v}(\mathrm{B}, \mathrm{D})$ acetate before radial diameter (C) or fungal dryweight (D) was measured. To ensure homologous integration of $f a c B$ in the complementation strain, strains were grown in the presence of pyrithiamine (PT, facB was reintroduced into the $\triangle f a c B$ strain using the PT-resistant marker gene) and hygromycin (HM, facB was deleted using the $\mathrm{HM}$ resistance marker gene) (B, C). Plate pictures (B) are representative for the average radial diameter shown in (C). Standard deviations represent the average of 3 biological replicates with ${ }^{* \star *} p$-value $<0.0001$ in a 2-way multiple comparisons ANOVA test, comparing the $\operatorname{facB}$ deletion strain to the WT strain.

Figure 2. FacB regulates acetate metabolism. A. Heat map depicting log2FC (fold changes) from the RNA-sequencing data of genes encoding enzymes required for acetate metabolism in the wild-type (WT) and $\triangle$ facB strains in the presence of $0.1 \% \mathrm{w} / \mathrm{v}$ or $1 \% \mathrm{w} / \mathrm{v}$ acetate after $0.5 \mathrm{~h}$ and $6 \mathrm{~h}$. The log2FC for the WT strain is based on the comparison of gene expression between the WT strain grown in fructose-rich medium and after transfer to acetate-rich medium; whereas log $2 \mathrm{FC}$ for the $\triangle \mathrm{facB}$ strain is from the comparison between the WT and $\mathrm{facB}$ deletion strain for each acetate condition. B. Validation of RNAsequencing data by qRT-PCR shows that FacB is required for the transcriptional expression of genes encoding enzymes involved in acetate metabolism. Strains were first grown in minimal medium (MM) supplemented with fructose before mycelia were transferred to acetate-containing MM, RNA was extracted and reverse-transcribed to cDNA and qRT-PCR was run on genes facA, Afu1g12340, Afu6g14100 and acuD. Gene expressions were normalised by $\beta$-tubulin. C. Results of Pearson Correlation Analysis between 1291 the RNA-sequencing and qRT-PCR datasets for 4 genes encoding enzymes 
1292 involved in acetate metabolism. Gene fold-change values were used for the

1293

1294

1295

1296

1297

1298

1299

1300

1301

1302

1303

1304

1305

1306

1307

1308

1309

1310

1311

1312

1313

1314

1315 analysis, which was carried out in Prism Graphpad ( ${ }^{*} p$-value $<0.05$, ${ }^{* *} p$-value $<$ 0.005). D. FacB is required for acetyl-CoA synthetase (ACS) and isocitrate lyase (ICL) activities. Strains were grown in fructose-rich MM for $24 \mathrm{~h}$, before mycelia were transferred to MM containing $1 \% \mathrm{w} / \mathrm{v}$ acetate for $0.5 \mathrm{~h}, 6 \mathrm{~h}$ and 22 $\mathrm{h}$ and total cellular proteins were extracted and enzyme activities were measured. Standard deviations represent the average of 3 biological replicates with ${ }^{* *} p$-value $<0.001,{ }^{* * *} p$-value $<0.0001$ in a 2 -way multiple comparisons ANOVA test when comparing the facB deletion strain to the WT strain.

\section{Figure 3. Acetate utilisation is subject to carbon catabolite repression} (CCR). A. - B. Expression of genes facA, Afu1g12340, Afu6g14100 and acuD, as determined by qRT-PCR, in strains grown for $24 \mathrm{~h}$ in minimal medium (MM) supplemented with fructose and then transferred for $0.5 \mathrm{~h}$ to MM supplemented with either acetate or acetate and glucose. Graphs in panel A. show results from growth in $12.2 \mathrm{mM}$ for each carbon source whereas graphs in panel B. show results from growth in $122 \mathrm{mM}$ for each carbon source. C. Activities of acetyl-CoA synthetase (ACS) and isocitrate lyase (ICL) in strains incubated for $0.5 \mathrm{~h}, 6 \mathrm{~h}$ and $22 \mathrm{~h}$ in MM supplemented with $122 \mathrm{mM}$ acetate and $122 \mathrm{mM}$ glucose. Strains were first grown for $24 \mathrm{~h}$ in fructose MM before mycelia were transferred to acetate and glucose-containing MM. D. Percentage of residual glucose in supernatants of strains grown for $24 \mathrm{~h}$ in fructose-rich $\mathrm{MM}$ and after transfer to glucose MM for a total time period of $22 \mathrm{~h}$. Standard deviations represent the average of 3 biological replicates with ${ }^{*} p$-value $<0.01,{ }^{* *} p$-value $<$ $0.001,{ }^{* * *} p$-value $<0.0001$ in a 2 -way multiple comparisons ANOVA test when 
1316

comparing the $f a c B$ deletion strain to the WT strain or when comparing the WT strain in two conditions (indicated by a line).

\section{Figure 4. The extracellular carbon source affects the levels of secreted} secondary metabolites (SMs). A, B. Heat map of the log2 fold-change (FC), as determined by RNA seq, of genes predicted to encode enzymes required for SM biosynthesis in the wild-type (WT) and $\triangle$ facB strains when grown for $0.5 \mathrm{~h}$ and $6 \mathrm{~h}$ in the presence of $0.1 \% \mathrm{w} / \mathrm{v}$ or $1 \% \mathrm{w} / \mathrm{v}$ acetate or when comparing gene expression in the WT strain in the presence of different acetate concentrations and in the presence of fructose (control, CTRL condition). In grey, are genes that did not show a significant FC. C. Quantities of identified SMs, as determined by high performance liquid chromatography (HPLC), in the WT and $\triangle f a c B$ strains when grown for $24 \mathrm{~h}$ in minimal medium supplemented with $0.1 \%$ $\mathrm{w} / \mathrm{v}($ LGLU $=$ low glucose; LACET $=$ low acetate $)$ or $1 \% \mathrm{w} / \mathrm{v}$ glucose $(\mathrm{HGLU}=$ high glucose; HACET = high acetate) or acetate. SM quantities were normalised by fungal dry weight (DW). Standard deviations represent the average of 4 biological replicates with ${ }^{*} p$-value $<0.01,{ }^{* *} p$-value $<0.001,{ }^{* * *} p$ value $<0.0001$ in a 2-way multiple comparisons ANOVA test.

\section{Figure 5. Acetate utilisation impacts cell wall polysaccharide content,} oxidative stress, caspofungin and immune cell resistance in A. fumigatus. A, B. Percentage of total (A.) and individual (B.) sugars identified in the alkaliinsoluble (AI) and alkali-soluble (AS) fractions by gas liquid chromatography of the WT strain when grown for $24 \mathrm{~h}$ in MM supplemented with glucose and acetate. Standard deviations represent the average of 4 biological replicates and ${ }^{* * *} p$-value $<0.0001$ in a 2 -way multiple comparisons ANOVA test when comparing the acetate condition to the glucose condition. C. The wild-type (WT) 
1341 strain was grown from $10^{5}$ spores for 5 days on glucose (GMM) or acetate minimal medium (AMM) supplemented with different concentrations of oxidative stress-inducing compounds. Colony diameters were measured and normalised by the control condition and expressed as percentage of growth in comparison to the control condition.

D. As described in C., with the exception that GMM or AMM was supplemented with increasing concentrations of caspofungin. Standard deviations represent the average of 3 biological replicates and ${ }^{* *} p$ value $<0.001,{ }^{* *} p$-value $<0.0001$ in a 2 -way multiple comparisons ANOVA test comparing the acetate condition to the glucose condition. E. The WT strain was pre-grown for $8 \mathrm{~h}$ or $13 \mathrm{~h}$ in GMM or AMM respectively, before hyphae were incubated with different concentrations of human neutrophils for $1 \mathrm{~h}$. Subsequently, cells were lysed and hyphal viability was assessed via an MTT assay and calculated. Standard deviations represent the average of 3 biological replicates and ${ }^{*} p$-value $<0.05$ in a one-tailed $t$-test, comparing the acetate condition to the glucose condition. F, G. Murine bone marrow-derived macrophage (BMDM) phagocytosis (F.) and killing (G.) of $A$. fumigatus conidia. BMDMs were incubated with fungal conidia before they were stained with calcofluor white and the percentage of phagocytosed conidia was assessed by microscopy and calculated. To assess fungal viability, conidia-macrophage mixtures were lysed, diluted and inoculated on plates containing complete medium before colony forming units (CFU) were assessed and percentage of viability calculated. Standard deviations represent the average of 3 biological replicates and ${ }^{*} p$-value $<0.05,{ }^{* *} p$-value $<0.005$ in a paired t-test. invasive aspergillosis. Survival curves ( $n=10 /$ strain and $n=5$ for control) of 
1366 Galleria melonella (A.) and mice (B.) infected with the respective $A$. fumigatus 1367 strains. Phosphate buffered saline (PBS) without conidia was given as a negative control. Indicated P-values are based on the Log-rank, Mantel-Cox and Gehan-Breslow-Wilcoxon tests comparing the facB deletion strain to the WT and facB complemented strains. Fungal burden in murine lungs after 3 (C.) and 7 (D.) days post-infection (p.i.) with the different $A$. fumigatus strains. Murine lungs were excised, ruptured and re-suspended, before dilutions were prepared that were incubated on plates containing complete medium. Fungal growth was assessed by counting the colony forming units (CFU) on the plates for each dilution. Inflammation in murine lungs after 3 (E.) days post-infection (p.i.) with the different $A$. fumigatus strains. Murine lungs were excised and slides of lung sections were prepared. To quantify lung inflammation of infected animals, inflamed areas on slide images were analysed using the thresholding tool in ImageJ software. Standard deviations represent the average of three biological replicates (lungs from different mice) with ${ }^{*} p$-value $<0.01,{ }^{* *} p$-value $<$ $0.001,{ }^{* * *} p$-value $<0.0001$ in a 2 -way multiple comparisons ANOVA test. $\mathbf{G}$. Histopathology of mice infected with the different $A$. fumigatus strains. Lungs were excised at 3 days post-infection (p.i.) before lung sections were prepared and stained with HE (Hematoxylin and Eosin) or with Grocott's methenamine silver (GMS). 
Table 1. Number of differentially expressed genes (DEGs, $-1<\log 2 \mathrm{FC}<1$ ) identified by minimal medium supplemented with 0.1 or $1.0 \% \mathrm{w} / \mathrm{v}$ acetate.

\begin{tabular}{|l|c|c|c|}
\hline \multicolumn{4}{|c|}{ Comparison acetate versus fructose in the wild-type strain } \\
\hline Condition & Up-regulated genes & Down-regulated genes & Total \\
\hline $0.1 \%$ acetate $0.5 \mathrm{~h}$ & $794(54.7 \%)$ & $658(45.3 \%)$ & 1452 \\
\hline $0.1 \%$ acetate $6.0 \mathrm{~h}$ & $1698(54 \%)$ & $1445(46 \%)$ & 3143 \\
\hline $1 \%$ acetate $0.5 \mathrm{~h}$ & $1107(47.8 \%)$ & $1211(52.2 \%)$ & 2318 \\
\hline $1 \%$ acetate $6.0 \mathrm{~h}$ & $882(66.3 \%)$ & $448(33.7 \%)$ & 1330 \\
\hline & Comparison $\Delta$ facB versus wild-type & 179 \\
\hline $0.1 \%$ acetate $0.5 \mathrm{~h}$ & $34(18.9 \%)$ & $145(81.1 \%)$ & 1306 \\
\hline $0.1 \%$ acetate $6.0 \mathrm{~h}$ & $710(54.3 \%)$ & $596(45.7 \%)$ & 678 \\
\hline $1 \%$ acetate $0.5 \mathrm{~h}$ & $54(28.6 \%)$ & $134(71.4 \%)$ & $198(29 \%)$ \\
\hline $1 \%$ acetate $6.0 \mathrm{~h}$ & $482(71.0 \%)$ & & \\
\hline
\end{tabular}

1392

1393

1394

1395

1396 
Table 2. Minimal inhibitory concentrations (MIC) of different antifungal drugs $(\mu \mathrm{g} / \mathrm{ml})$

1401 minimal medium (AMM). Shown is the average and standard deviations of three RPMI and GMM.

\begin{tabular}{|l|l|l|l|l|}
\hline Medium & Amphotericin B & Voriconazole & Itraconazole & Posaconazole \\
\hline RPMI & $3.33 \pm 1.15$ & $0.25 \pm 0.00$ & $0.42 \pm 0.14$ & $0.67 \pm 0.29$ \\
\hline GMM & $3.33 \pm 1.15$ & $0.33 \pm 0.14$ & $0.50 \pm 0.00$ & $1.00 \pm 0.00$ \\
\hline AMM & $1.17^{*} \pm 0.76$ & $0.21 \pm 0.07$ & $0.33 \pm 0.14$ & $0.67 \pm 0.29$ \\
\hline
\end{tabular}




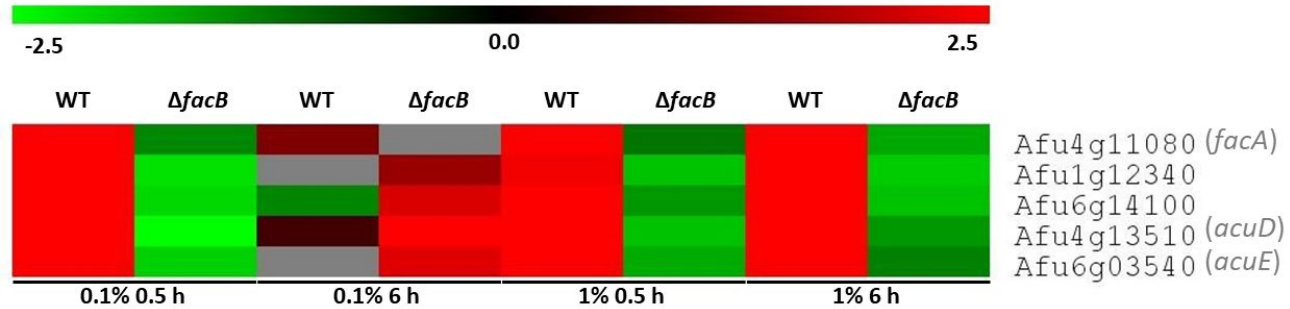

B.

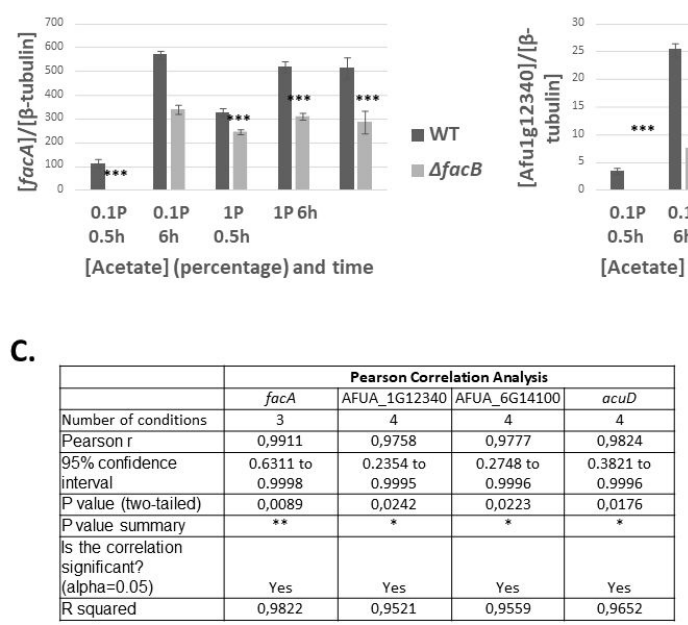

D.

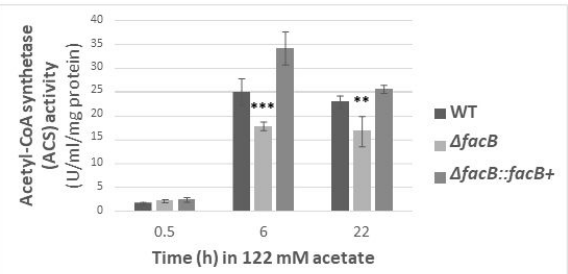

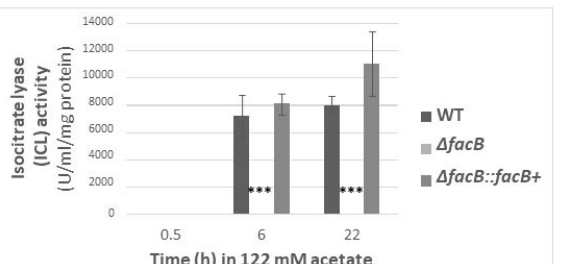

Time (h) in $122 \mathrm{mM}$ acetate 
A.

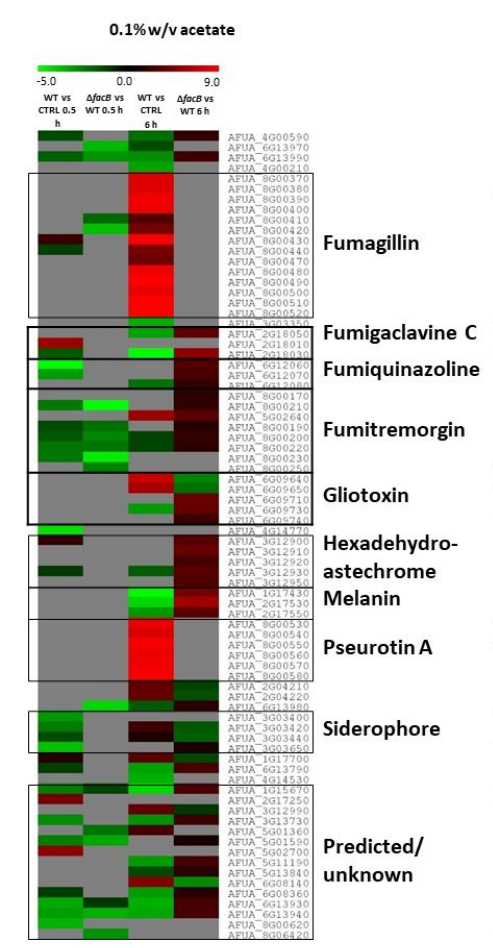

B.

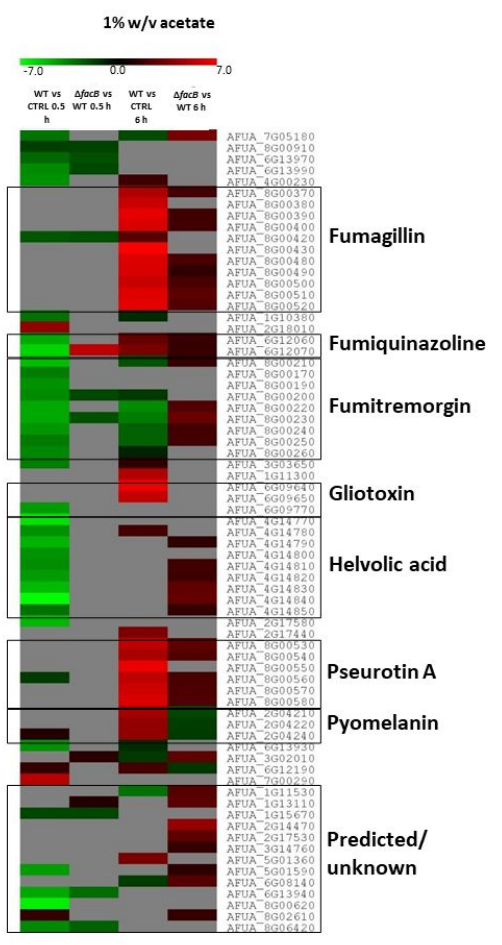

c.
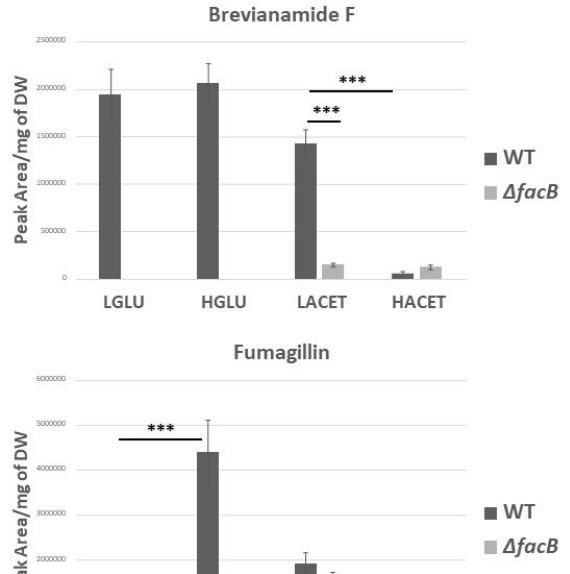

这势
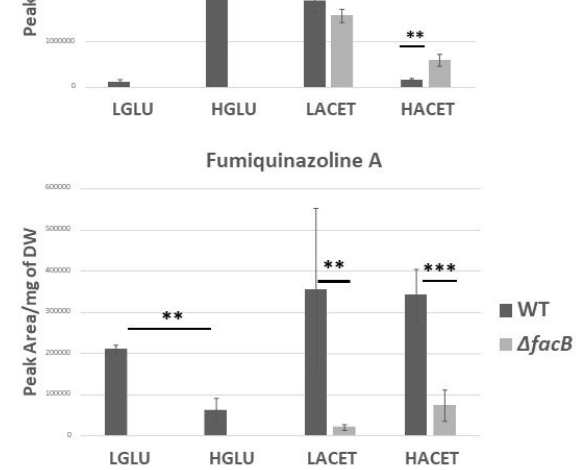

Pyripyropene A

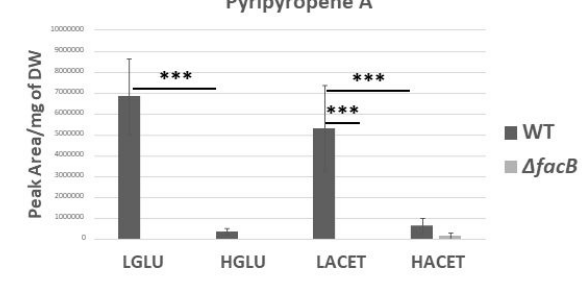

Fumitremorgin C

- WT

$\triangle f a c B$

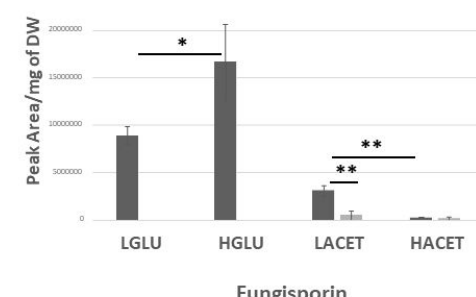

- W

$\Delta f a c B$
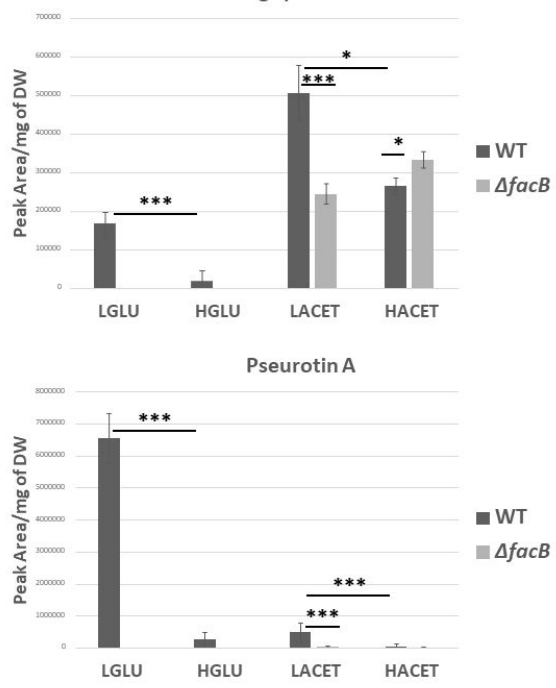

- WT

$\triangle f a c B$ 
A.

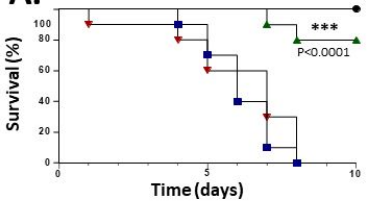

C.

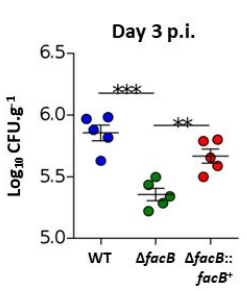

D.

B.

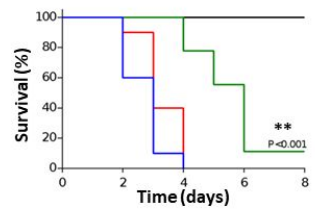

- Control

- Wild-type

- $\triangle$ facB

* $\triangle f a c B:: f a c B^{+}$

- Wild-type

- $\Delta f a c B$

- $\Delta f a c B:: f a c B^{+}$

40.001

E.

Day 7 p.i.

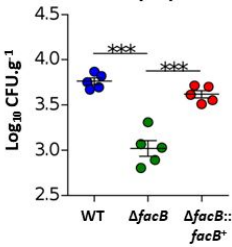

ns

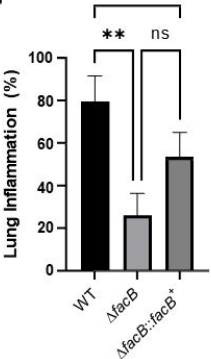

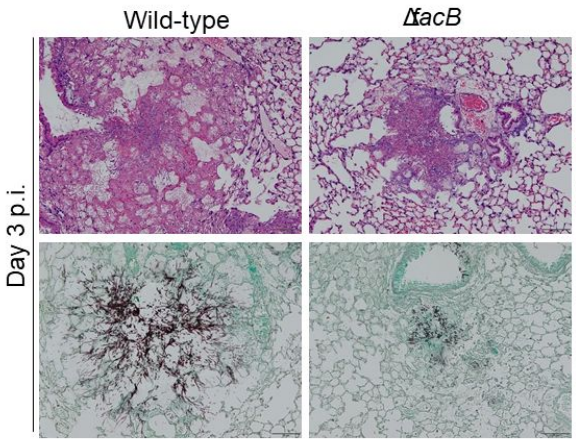

$\triangle f a c B:$ facB $^{+}$

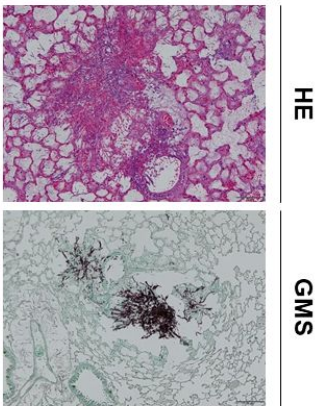

